\title{
A preliminary survey and range extension of millipedes species introduced in Brazil (Myriapoda, Diplopoda)
}

\author{
Luiz Felipe Moretti Iniesta ${ }^{1,2,6}$; Rodrigo Salvador Bouzan ${ }^{1,2,7}$; Patrícia Elesbão da Silva Rodrigues ${ }^{3}$; Thais Melo de Almeida ${ }^{4}$; \\ Ricardo 0tt ; Antonio Domingos Brescovit ${ }^{1,8}$
}

\footnotetext{
1 Instituto Butantan, Laboratório Especial de Coleções Zoológicas (LECZ). São Paulo, SP, Brasil.

${ }^{2}$ Universidade de São Paulo (USP), Instituto de Biociências (IB-USP). São Paulo, SP, Brasil. ORCID: http://orcid.org/0000-0003-4448-3940.E-mail: patiesilva@yahoo.com.br

${ }^{4}$ Instituto Nacional de Pesquisas da Amazônia (INPA), Coordenação de Biodiversidade (CBIO), Laboratório de Sistemática e Ecologia de Invertebrados do Solo (LSEIS). Manaus, AM, Brasil. ORCID: http://orcid.org/0000-0002-0282-1043. E-mail: thais.mel020@gmail.com

5 Secretaria do Meio Ambiente e Infraestrutura (SEMA), Museu de Ciências Naturais. Porto Alegre, RS, Brasil. ORCID: http://orcid.org/0000-0001-7392-1415. E-mail: rott@fzb.rs.gov.br

${ }^{6}$ ORCID: http://orcid.org/0000-0002-0529-4162. E-mail: luiz-moretti@hotmail.com (corresponding author)

7 ORCID: http://orcid.org/0000-0002-5331-7031. E-mail: rodrigobouzan@outlook.com

${ }^{8}$ ORCID: http://orcid.org/0000-0002-1511-5324. E-mail: antonio.brescovit@butantan.gov.br
}

${ }^{3}$ Universidade do Vale do Rio dos Sinos (UNISINOS), Laboratório de Diversidade e Sistemática de Arachnida (LADISA). São Leopoldo, RS, Brasil.

\begin{abstract}
The present study provides historical and new records of the introduced millipedes species in Brazil, Oxidus gracilis (C.L. Koch, 1847), Orthomorpha coarctata (Saussure, 1860) (Paradoxosomatidae), Prosopodesmus jacobsoni Silvestri, 1910 (Haplodesmidae), Trachyjulus calvus (Pocock, 1893a), Glyphiulus granulatus (Gervais, 1847) (Cambalopsidae), Trigoniulus corallinus (Gervais, 1842), Leptogoniulus sorornus (Butler, 1876), Epitrigoniulus cruentatus (Brölemann, 1903) (Pachybolidae), Paraspirobolus lucifugus (Gervais, 1837) (Spirobolellidae), Cylindroiulus britannicus (Verhoeff, 1891), Cylindroiulus truncorum (Silvestri, 1896) (Julidae), and Rhinotus purpureus (Pocock, 1894) (Siphonotidae). Among the 27 federative units in Brazil, 21 states present at least one record of a non-native species. Orthomorpha coarctata was the most widely distributed species, occurring in 15 states. Glyphiulus granulatus (state of Rio Grande do Sul), C. truncorum (São Paulo), and R. purpureus (Amazonas) were recorded from only one Brazilian state. The Southeast region concentrates most of the compiled records $(42,6 \%)$ and richness by grid (5-7 species), mainly in urban areas of the states of Rio de Janeiro and São Paulo.
\end{abstract}

Keywords. Invasive species; Synanthropic millipedes; Schubart; Oxidus gracilis; Orthomorpha coarctata.

\section{INTRODUCTION}

Members of the class Diplopoda are distributed on all continents, excepting the Antarctica (Hoffman, 1980; Golovatch \& Kime, 2009). Millipedes are commonly observed on tropical, subtropical, and temperate forests (Golovatch \& Kime, 2009), performing important ecological roles as detritivorous and biogeographical indicators (Schubart, 1942b; Crawford, 1992; Golovatch \& Kime, 2009; Suzuki et al., 2013; Nsengimana et al., 2018; Potapov et al., 2019).

Introduced species are widely accepted as one of the main direct causes of biodiversity loss and habitat alteration (Didham et al., 2005). In addition, non-native species have been reported as widely introduced around the world by human activities such as gardening, cultivation of plants, and soil transport. Numerous studies have reported the presence of millipedes in man-made habitats (Vicente \& Enghoff, 1999; Golovatch \& Kime, 2009; Shelley \& Golovatch, 2011), including well-established populations in urban and rural areas (Hopkin \& Read, 1992; Korsós et al., 2002; Bogyó et al., 2015). Some millipedes have been reported as pests causing significant economic damage (Butcher, 1936; Kuria \& Eijnatten, 1981; Brunke et al., 2012). In Brazil, some introduced millipedes are considered agricultural pests in polyor monocultures based on observations of immatures and adults feeding on seedlings, tubers, and fruits (Schubart, 1942b; Boock \& Lordello, 1952; Lordello, 1954). Importantly, the identification of a given species as introduced depends on the 
availability of taxonomic data across large geographical regions. However, except for descriptive papers and taxonomic revisions made mainly by the authors Schubart, Brölemann, and Hoffman in the last century, there is no an updated list of millipede species for Brazil.

Based on a substantial material of millipedes from Brazilian collections, new records of non-native species were detected. In this perspective, to promote further studies regarding the millipedes in the country, the current study presents a preliminary survey of introduced millipedes species and their distribution. Maps for each species and comments on their identifications are provided here.

\section{MATERIAL AND METHODS}

The occurrence data for the introduced species was extracted from the literature and material deposited in the following institutions (curators in parentheses): IBSP, Instituto Butantan, São Paulo (A.D. Brescovit); INPA, Instituto Nacional de Pesquisas da Amazônia, Manaus (M.L. de Oliveira); MCN, Museu de Ciências Naturais, SEMA, Porto Alegre (R.Ott); MCTP, Museu de Ciências eTecnologia da Pontifícia Universidade Católica do Rio Grande do Sul, Porto Alegre (R. Teixeira); $\mathbf{M H N C l , ~ M u s e u ~ d e ~ H i s t o ́ r i a ~}$ Natural do Capão do Imbuia, Curitiba (V. Abilhoa); MNRJ, Museu Nacional, Universidade Federal do Rio de Janeiro, Rio de Janeiro (A.B. Kury); MPEG, Museu Paraense Emílio Goeldi, Belém, Pará (A.B. Bonaldo); MZ/Unisinos, Museu de Zoologia da Unisinos, São Leopoldo, Rio Grande do Sul (E.N.L. Rodrigues); MZSP, Museu de Zoologia, Universidade de São Paulo (R. Pinto-da-Rocha); UFMT, Universidade Federal do Mato Grosso, Cuiabá (A. Chagas-Jr.).

Morphological observations and drawings were made using a Leica MZ 12 stereomicroscope. Photographs were taken with a Leica DFC 500 digital camera mounted on a Leica MZ 16A stereomicroscope and extended focal range images were composed with Leica Application Suite version 2.5.0, at IBSP. The specimens are preserved in $70 \%$ or $80 \%$ ethanol. The determination of the species was based on the descriptive notes and revisions performed by Schubart (1942c, 1946a, b), Mauriès (1980), Blower (1985), Korsós \& Enghoff (1990), Shelley \& Lehtinen (1999), Likhitrakarn et al. (2011), Golovatch et al. (2007, 2012), Wesener (2014) and Nguyen et al. (2017). All records obtained are mapped in the Figs. 1-2 and Table 1.

The geographical coordinates and collection data were obtained from the original literature (when provided) or the labels for each specimen consulted. We used the centroids of the municipalities when the exact locality was not provided. The data of the species are noted for each taxon according to the following pattern: Material examined: State: County, locality [geographical coordinates], data, collector (voucher); Historical records: State: County, locality [geographical coordinates], data, collector (reference consulted). The records gathered from the literature were compiled with all information provided by their respective authors, although some of these records only the localities were informed.

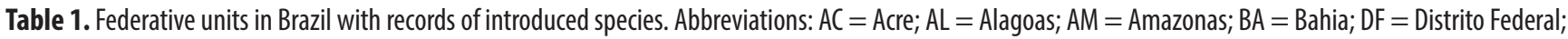
$\mathrm{ES}=$ Espírito Santo; $\mathrm{GO}=$ Goiás; $\mathrm{MT}=$ Mato Grosso; $\mathrm{MS}=$ Mato Grosso do Sul; $\mathrm{MG}=$ Minas Gerais; $\mathrm{PA}=$ Pará; $\mathrm{PB}=$ Paraíba; $\mathrm{PR}=\mathrm{Paraná} ; \mathrm{PE}=\mathrm{Pernambuc0} ; \mathrm{RJ}=$ Rio de Janeiro; RS = Rio Grande do Sul; RO = Rondônia; RR = Roraima; $S C=$ Santa Catarina; $S P=$ São Paulo; $T 0=$ Tocantins.

\begin{tabular}{|c|c|c|c|c|c|c|c|c|c|c|c|c|c|c|c|c|c|c|c|c|c|}
\hline & \multicolumn{6}{|c|}{ North } & \multicolumn{4}{|c|}{ Northeast } & \multicolumn{4}{|c|}{ Central-West } & \multicolumn{4}{|c|}{ Southeast } & \multicolumn{3}{|c|}{ South } \\
\hline & $\mathbf{R R}$ & AM & PA & $A C$ & RO & TO & PB & PE & AL & BA & DF & GO & MT & MS & MG & ES & RJ & SP & PR & SC & RS \\
\hline \multicolumn{22}{|l|}{ Order Polydesmida } \\
\hline \multicolumn{22}{|l|}{ Paradoxosomatidae } \\
\hline Oxidus gracilis (C.L. Koch, 1847) & & + & & & & & & & & & + & & & + & + & + & + & + & + & + & + \\
\hline Orthomorpha coarctata (Saussure, 1860) & & + & + & + & + & + & + & + & + & & & + & + & & + & + & + & + & + & & \\
\hline \multicolumn{22}{|l|}{ Haplodesmidae } \\
\hline Prosopodesmus jacobsoni Silvestri, 1910 & & & & & & & & & & & & & & & & & + & + & & & \\
\hline \multicolumn{22}{|l|}{ Order Spirostreptida } \\
\hline \multicolumn{22}{|l|}{ Cambalopsidae } \\
\hline Trachyjulus calvus (Pocock, 1893) & & & & & & & & & & + & & & & & & & + & & & & \\
\hline Glyphiulus granulatus (Gervais, 1847) & & & & & & & & & & & & & & & & & & & & & + \\
\hline \multicolumn{22}{|l|}{ Order Spirobolida } \\
\hline \multicolumn{22}{|l|}{ Pachybolidae } \\
\hline Trigoniulus corallinus (Gervais, 1841) & + & + & + & & & + & & + & & & & & & & & & + & & & & \\
\hline Leptogoniulus sorornus (Butler, 1876) & & & & & & & & & & + & & & & & & + & + & & & & \\
\hline Epitrigoniulus cruentatus (Brölemann, 1903) & & + & + & & & & & & & & & & & & & & & & & & \\
\hline \multicolumn{22}{|l|}{ Spirobolellidae } \\
\hline Paraspirobolus lucifugus (Gervais, 1837) & & & & & & & & & & & & & & & & + & + & + & & & \\
\hline \multicolumn{22}{|l|}{ Order Julida } \\
\hline \multicolumn{22}{|l|}{ Julidae } \\
\hline Cylindroiulus britannicus (Verhoeff, 1891) & & & & & & & & & & & & & & & & & + & + & & & \\
\hline Cylindroiulus truncorum (Silvestri, 1896) & & & & & & & & & & & & & & & & & & + & & & \\
\hline \multicolumn{22}{|l|}{ Order Polyzoniida } \\
\hline Siphonotidae & & & & & & & & & & & & & & & & & & & & & \\
\hline Rhinotus purpureus (Pocock, 1894) & & + & & & & & & & & & & & & & & & & & & & \\
\hline
\end{tabular}




\section{RESULTS}

\section{Order Polydesmida Family Paradoxosomatidae}

\section{Oxidus gracilis (C.L. Koch, 1847)} (Figs. 1A, 4B, 5A)

Descriptive notes: See Nguyen et al. (2017: 6, figs. 1-2).

Note: Based on examined material, immatures and females of $O$. gracilis cannot be identified and morphologically distinguished from those of Orthomorpha coarctata.

Identification: The species can be easily distinguished from autochthones Neotropical paradoxosomatids mainly by gonopod features (Fig. 5A). Males of 0 . gracilis are recognized by femorite strongly expanded distally; postfemoral spine pointed tuberculiform; postfemoral process lamellar and bent upwards from midpart, serrated at distolateral portion; solenophere with mesal lobe well-developed (see Nguyen et al., 2017).

Distribution: Oxidus Cook, 1911 occurs in the SE Asia (Jeekel, 1968; Nguyen et al., 2017), while the species 0 . gracilis is widely distributed around the world due to commercial activities (Nguyen \& Sierwald, 2013), occurring in USA and Hawaii (Shelley et al., 1998), Europe (Blower, 1985), and Asia (Korsós, 2004; Nguyen et al., 2017). The species is considered urban and agricultural pest (O’Neill \& Reichle, 1970). In Brazil, the species presents a large distribution range, occurring in urban and
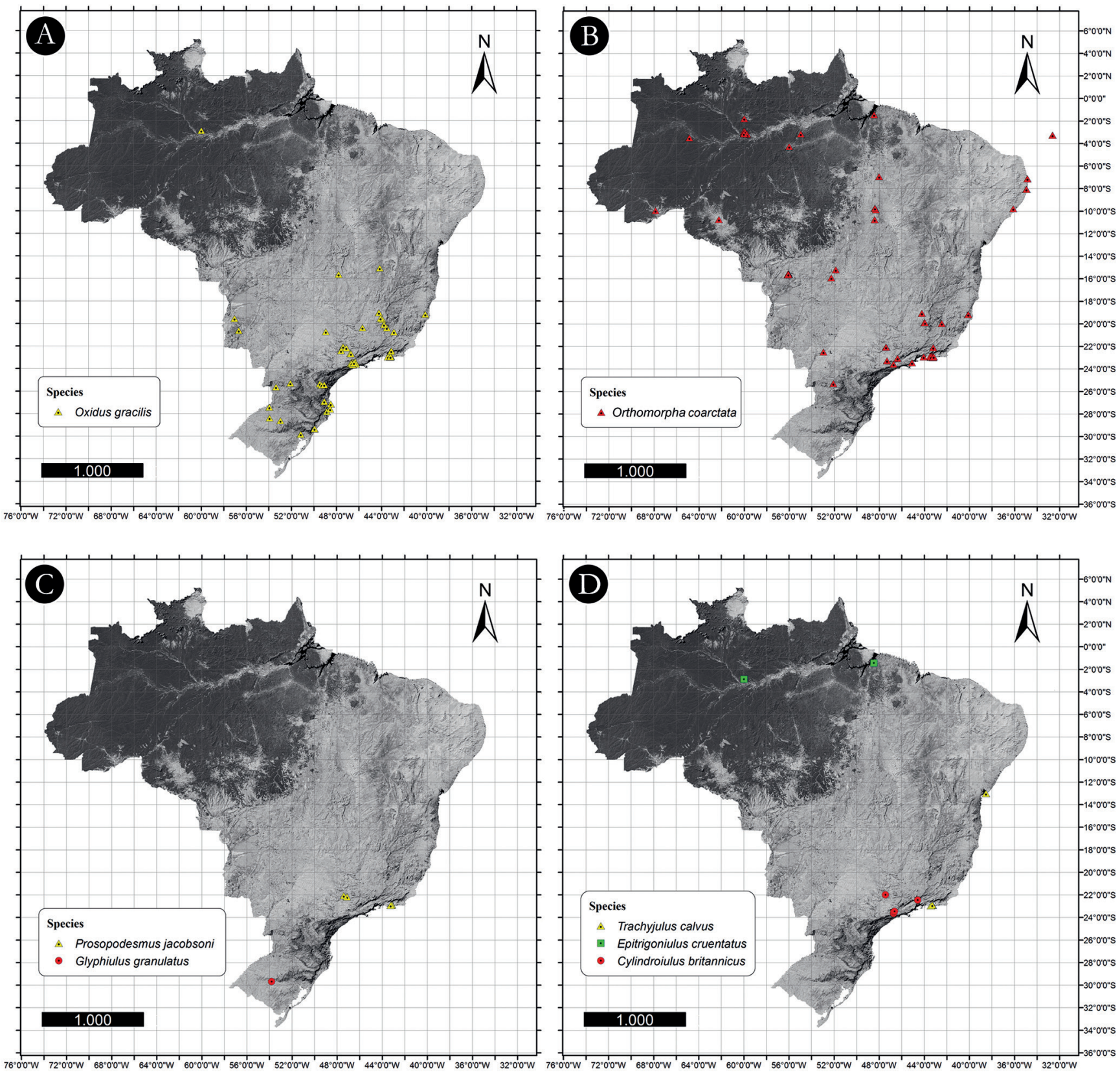

Figure 1. Distribution maps of Oxidus gracilis, Orthomorpha coarctata (Polydesmida, Paradoxosomatidae), Prosopodesmus jacobsoni (Polydesmida, Haplodesmidae), Glyphiulus granulatus (Spirostreptida, Cambalopsidae), Trachyjulus calvus (Spirostreptida, Cambalopsidae), Epitrigoniulus cruentatus (Spirobolida, Pachybolidae), and Cylindroiulus britannicus (Julida, Julidae). 
rural areas, greenhouses, caves, and in forests partially preserved (Iniesta et al., 2020).

Material examined: Amazonas: Manaus [-03.063877 ${ }^{\circ} \mathrm{S}$; $-60.036493^{\circ} \mathrm{W}$, Embrapa Amazônia Ocidental, banana plantation, near the Climatology laboratory, 28.iv.2016, T.M. Almeida \& A.E.C. Silveira coll., $120^{\prime} 892$ immatures (INPA); Distrito Federal: Sobradinho [-15.658554 ${ }^{\circ} \mathrm{S}$; $-47.791846^{\circ} \mathrm{W}$ ], cave Face Leste, 26.vi.2013, M.P. Bolfarini coll., 207 (IBSP 5509); Mato Grosso do Sul: Corumbá [-19.577553 $\left.{ }^{\circ} \mathrm{S} ;-57.037810^{\circ} \mathrm{W}\right]$, Passo do Lontra, 2000, J. Raizer coll., o' (IBSP 1035); Bodoquena [-20.631661 ${ }^{\circ} \mathrm{S}$; $-56.652781^{\circ} \mathrm{W}$, cave Califórnia, 17.iii.2001, C.S. Escarpinati coll., or (IBSP 4276); ᄋ (IBSP 4282); ᄋ (IBSP 4270); ㅇ (IBSP 4271); ᄋ (IBSP 4277); 오 (IBSP 4280); Minas Gerais: Pains [-20.383591 ${ }^{\circ} \mathrm{S}$; $-45.661936^{\circ} \mathrm{W}$ ], cave Piriás, 30.i.2001, R. Ferreira coll., 20²우 (IBSP 3602);

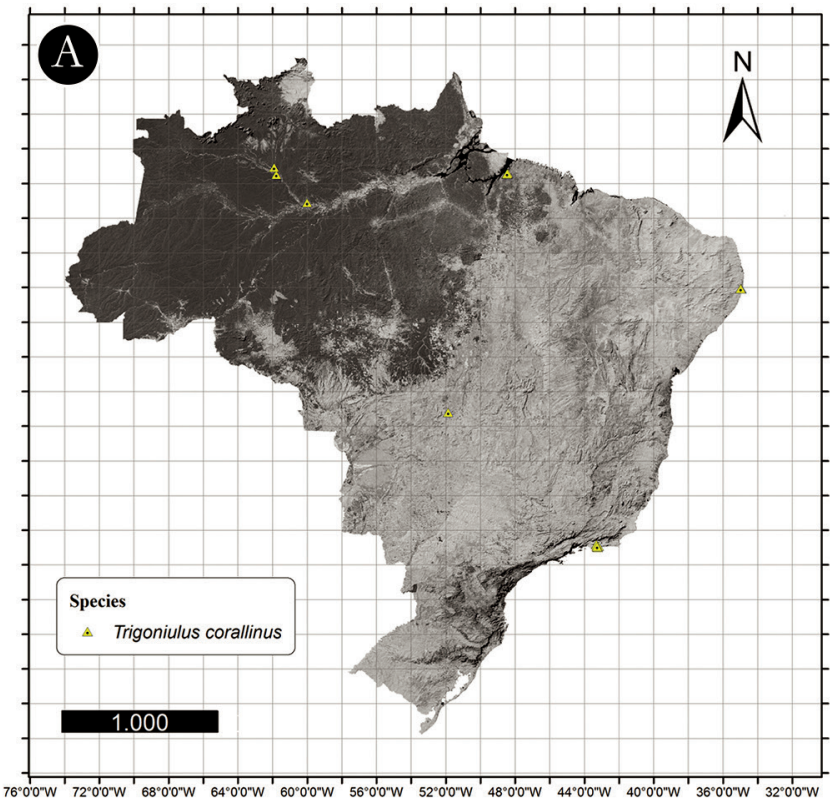

27.i.2000, Ferreira coll., $50^{*} 29$ (IBSP 3601); Itacarambi [-15.092057 ${ }^{\circ}$; $\left.-44.133344^{\circ} \mathrm{W}\right]$, Vilarejo Fabião II, 05.vii.2011, J.P.P. Pena-Barbosa coll., o' $0^{7}$ (IBSP 3726); Cordisburgo $\left[-19.050250^{\circ} \mathrm{S} ;-44.209663^{\circ} \mathrm{W}\right]$, Morena cave, 12-15.x.2007, ơ ㅇ (IBSP 3558); ơ 우 (IBSP 3556); 우 (IBSP 3559); 9 (IBSP 3557); Lagoa Santa [-19.635088 $\mathrm{S}$; $-43.898478^{\circ} \mathrm{W}$ ], 21.x.1947, O. Schubart coll., $50^{\prime} 39$ immatures (MZSP); Viçosa [-20.756373 ${ }^{\circ}$; $-42.883016^{\circ} \mathrm{W}$, ESAV, 14.x.1947, O. Schubart coll., 20" (MZSP); Ouro Preto $\left[-20.395727^{\circ} \mathrm{S} ;-43.502578^{\circ} \mathrm{W}\right], 17 . x .1947, \mathrm{O}$. Schubart coll., 40 69 1 immature (MZSP); Rio Acima [-20.088882 $\left.{ }^{\circ} \mathrm{S} ; \quad-43.791390^{\circ} \mathrm{W}\right]$, cave 0020_VG43, 02-10.viii.2011, R. Andrade coll., ox 우 (IBSP 6622); 20' 9 (IBSP 6671); cave SPD_38, 25-27.vii.2013, Bessi et al., coll., $0^{x}$ ㅇ (IBSP 7150); ㅇ (IBSP 7149); Matozinhos [-19.565584 ${ }^{\circ}$; $\left.-44.078665^{\circ} \mathrm{W}\right]$, cave LF_22, 16.viii-14. ix.2017, Eq. Spelayon coll., 20² 2 (IBSP 7473); Espírito
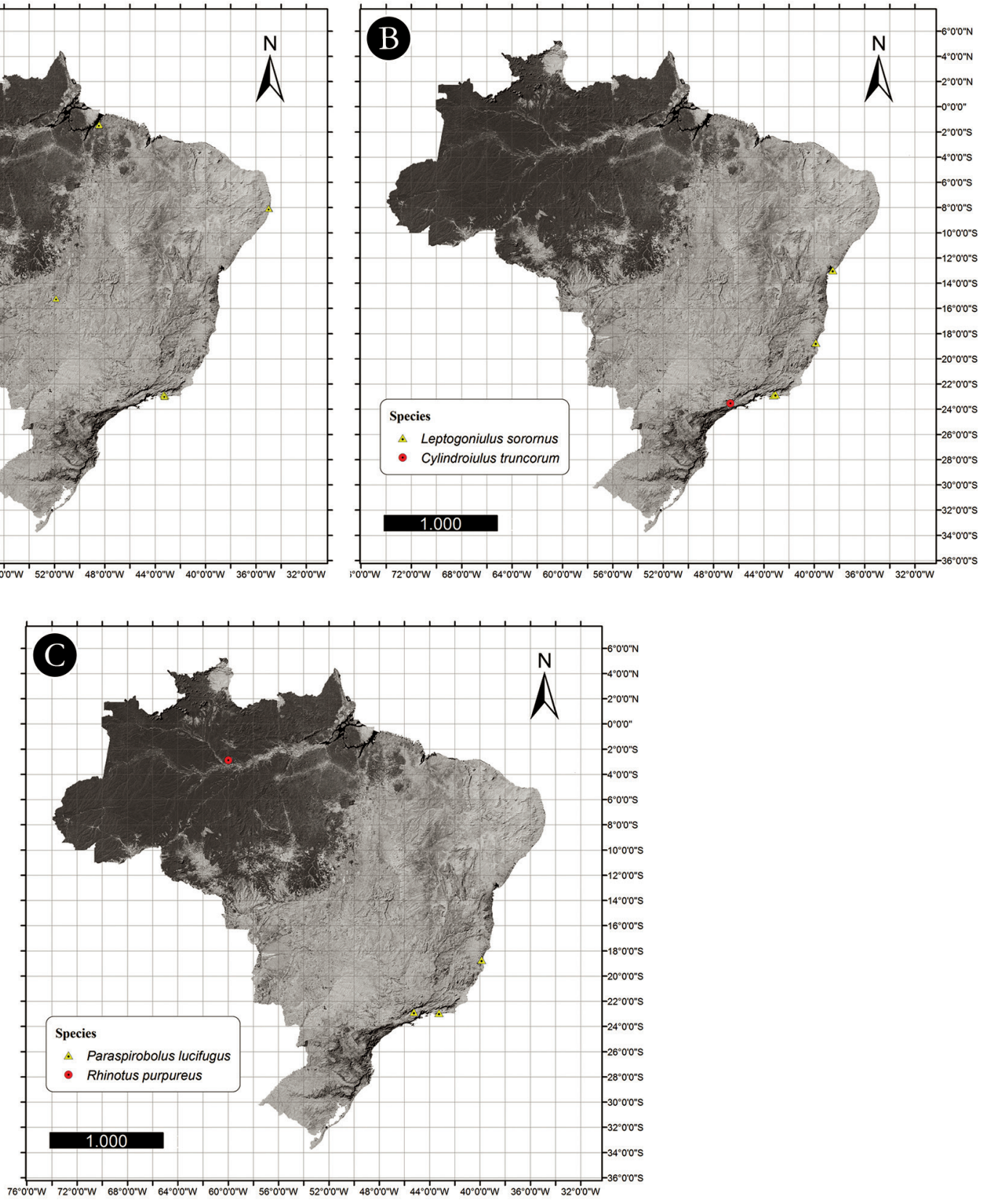

Figure 2. Distribution maps of Trigoniulus corallinus, Leptogoniulus sorornus (Spirobolida, Pachybolidae), Cylindroiulus truncorum (Julida, Julidae), Paraspirobolus lucifugus (Spirobolida, Spirobolellidae), and Rhinotus purpureus (Polyzoniida, Siphonotidae). 
Santo: Sooretama $\left[-19.1979,{ }^{\circ}\right.$; $\left.-40.0913^{\circ} \mathrm{W}\right], 24 . i i .2011$, A.F.R. Teixeira coll., 29 (MCN); Rio de Janeiro: Rio de Janeiro [-22.921765 ${ }^{\circ}$; $\left.-43.169510^{\circ} \mathrm{W}\right]$, O. Schubart coll., 18 immatures (MNRJ 11694); ㅇ (MNRJ 11740); $71 \mathrm{im}$ matures (MNRJ 11749); (MNRJ 11728); (MNRJ 11732); (MNRJ 11746); Petrópolis [-22.921765 ${ }^{\circ}$; $\left.-43.169510^{\circ} \mathrm{W}\right]$, O. Schubart coll., o (MNRJ 11733); 22 immatures (MNRJ 11737); São Paulo: Arujá [-22.668644 ${ }^{\circ}$; $-46.320865^{\circ} \mathrm{W}$ ], 06.xii.1995, Vigilância Sanitária Guarulhos coll., $0^{x}$ \% (IBSP 944); Guarulhos [-23.678875 ${ }^{\circ}$; $-46.543425^{\circ} \mathrm{W}$ ], 28.ii.2002, Prefeitura Municipal de Guarulhos coll. (IBSP 903); Olímpia $\left[-20.741815^{\circ}\right.$; $-48.911570^{\circ}$ W], xi.1916, E. Garb coll., ơ (MZSP); Santo André $\left[-23.678234^{\circ} \mathrm{S} ;-46.543425^{\circ} \mathrm{W}\right], \quad x .1939, \quad$ B.L. Bastiani coll., 20`39 (IBSP 28); São Paulo [-23.567300' $-46.633047^{\circ} \mathrm{W}$ ], 01.i.1956, J. Becker coll., $20^{*} 3$ (MNRJ); $20^{\prime \prime} 29$ (IBSP 34); iv.1939, F. Paes de Barros coll., $20^{\star 1} 5$ ㅇ (IBSP 34); vii.1940, W. Bücherl coll., 40 $\$$ (IBSP 23); Tremembé $\left[-23.464143^{\circ} \mathrm{S}^{\prime}-46.625706^{\circ} \mathrm{W}\right], 10 . x .2001$, L. Goes \& L. Silva coll., \& (MNRJ); Santana [-23.501011 ${ }^{\circ} \mathrm{S}$; -46.626233 W], 08.x.1998, Centro Zoonoses Prefeitura Municipal de São Paulo coll., ơ 3 ? (IBSP 678); Vila Gomes [-23.576975 ${ }^{\circ}$; $-46.732075^{\circ} \mathrm{W}$ ], 26.viii.1998, F. Henrique

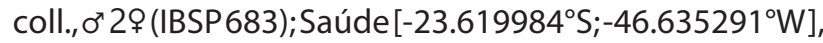
14.xi.2000, P. Kitamoto coll., $100^{7} 1291$ immature (IBSP 825); Jardim Rizzo [-23.572008 ${ }^{\circ}$; $\left.-46.732739^{\circ} \mathrm{W}\right]$, 20.xi.1998, Equipe IBSP coll., ơ (IBSP 673); $0^{7} \%$ (IBSP 672); \% (IBSP 675); Mogi das Cruzes, Parque São Martinho [-23.614280 ${ }^{\circ}$; $\left.-46.236089^{\circ} \mathrm{W}\right], 17 . x \mathrm{.} 2018$, R.S. Bouzan coll., $30^{7} 292$ immatures (IBSP 7757); Paraná: Piraquara
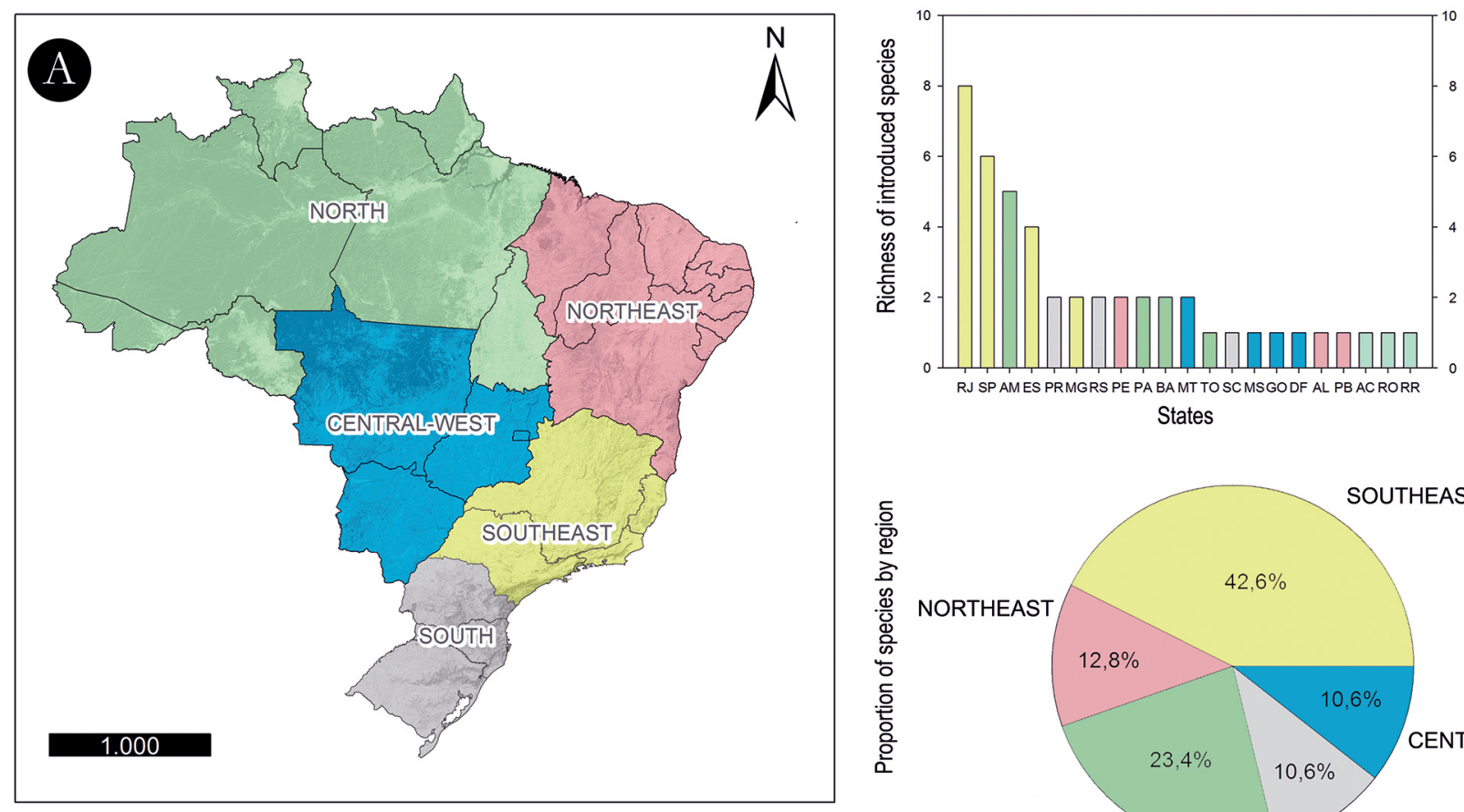

B
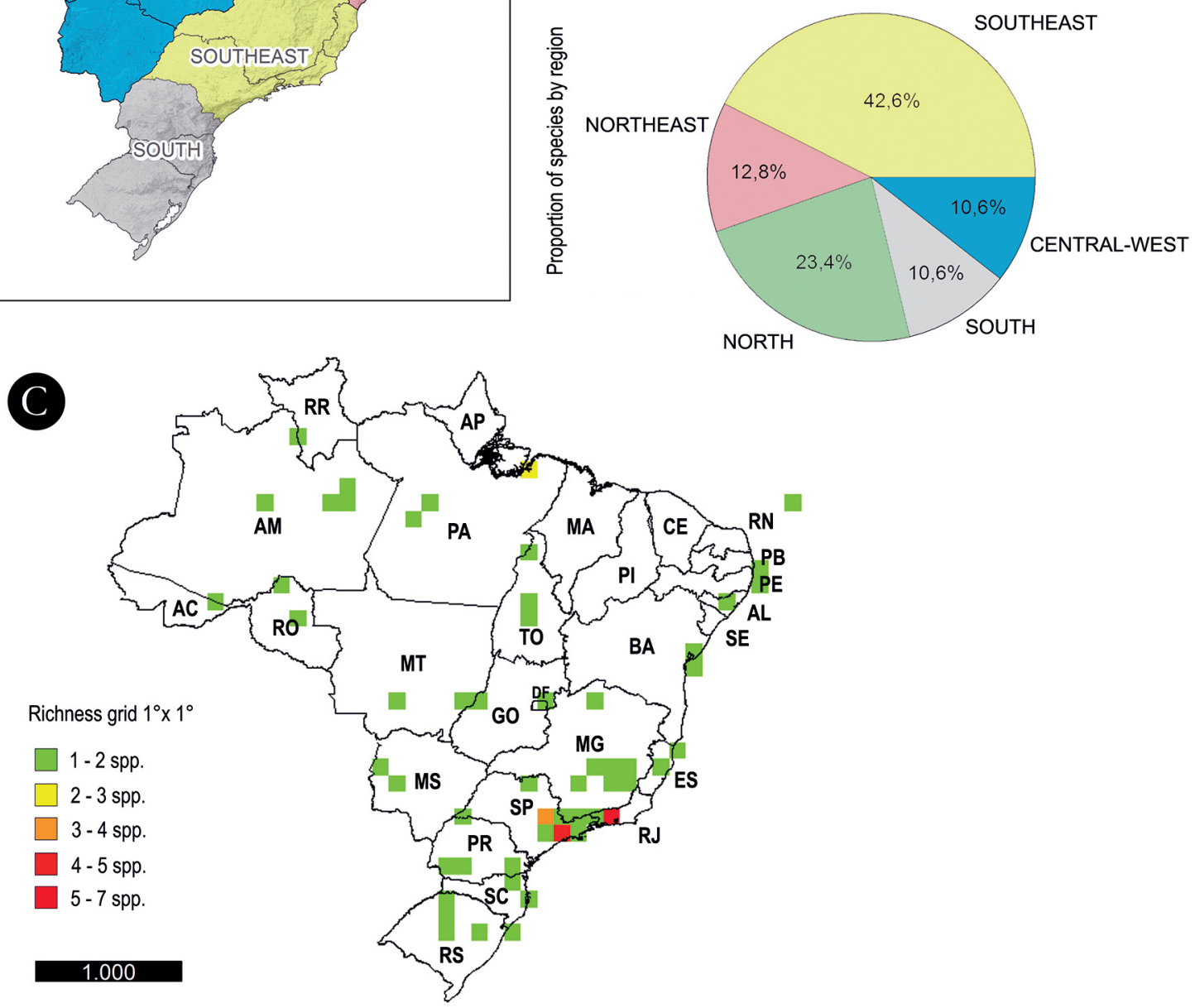

Figure 3. Introduced species in Brazil: (A) Brazilian regions; (B) Proportion of species by states and regions; (C) Richness by grid $1^{\circ} \times 1^{\circ}$. See Table 1 for the abbreviations of the states. 
[-25.454547 ${ }^{\circ}$; $\left.-49.062333^{\circ} \mathrm{W}\right]$, Banhado, 22.xii.2015, A. Chagas-Jr., M. Karam \& A. Kury coll., ơ 2 \% (UFMT); Nova Prata do Iguaçu $\left[-25.640129^{\circ} \mathrm{S} ;-53.345181^{\circ} \mathrm{W}\right]$, Salto Caxias, 24.ix.1990, R.S. Bérnils coll., ơ (MHNCl); Campo Magro [-25.369783 $\left.{ }^{\circ} \mathrm{S} ;-49.450466^{\circ} \mathrm{W}\right]$, cave Terra Boa, 27.vi.2015, W. Murguia coll., o' (IBSP 7405); Rio Grande do Sul: Selbach [-28.63129444º; -52.95194444W], xi.2016, C. Maldaner coll., 9 (INPA); Morrinhos do Sul [-29.3569 $\mathrm{S}$; $-49.91812^{\circ} \mathrm{W}$ ], 20.xii.2010, R. Ott coll., 30 7ㅇ (MCN 938); Sapucaia do Sul $\left[-29.840651^{\circ} \mathrm{S} ; \quad-51.147425^{\circ} \mathrm{W}\right]$, 07.xii.2012, E. Velinho coll., ơ 4오 (MCN 964); o' 50웅 (MCN 964); Três Passos [-27.453954 ${ }^{\circ}$; ; $-53.929651^{\circ} \mathrm{W}$ ], 14.ix.1947, A.C. Aguirre coll., ơ (MZSP); ljuí [-28.393218 S; $-53.920602^{\circ} \mathrm{W}$ ], 16.iv.1954, A.C. Aguirre coll., ơ (MZSP).

Historical records: Rio de Janeiro: Petrópolis [-22.921765 ${ }^{\circ}$; $\left.-43.169510^{\circ} \mathrm{W}\right]$, O. Schubart coll. (Schubart, 1945b); São Paulo: Amparo, Fazenda São Bento [-22.668644 $\left.{ }^{\circ} \mathrm{S} ;-46.737739^{\circ} \mathrm{W}\right], 08 . i i .1943, \mathrm{~F}$. Lane coll., 9o' 13ㅇ 1 immature (Schubart, 1945a); Mogi Guaçu [-22.185269 ${ }^{\circ}$; $\left.\quad-47.097326^{\circ} \mathrm{W}\right]$, 06.vii.1941, $60^{\prime \prime} 699$ immatures (Schubart, 1944); Monte Alegre

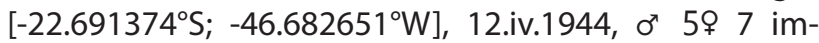
matures (Schubart, 1945a); Estação Experimental, 12.iv.1944, 130 7\% (Schubart, 1945a); Pirassununga [-22.067267º $\left.;-47.395011^{\circ} \mathrm{W}\right], 17 . i .1940,100^{\circ} 11 \% 5 \mathrm{im}-$ matures (Schubart, 1944); 03.iv.1940, 50 7926 immatures (Schubart, 1944); 22.v.1940, ox $\subsetneq 1$ immature (Schubart, 1944); 30.vii.1940, 50 5 क (Schubart, 1944); 13.ii.1941, ơ 4938 immatures (Schubart, 1945a); Fazenda São Domingos, 22.ix.1940, 160' 219 63 immatures (Schubart, 1945a); Fazenda Graciosa, 25.x.1940, 520'31우 21 immatures (Schubart, 1945a); Cachoeira, 23.xi.1940, \% (Schubart, 1944); 10.ii.1941, 20' 1 immature (Schubart, 1944); Fazenda Pedra Branca, 15.ii.1942, J. Gaspar

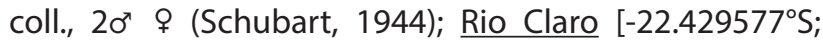
$-22.429577^{\circ} \mathrm{W}$ ], Fazenda São José, 26.ix.1941, 220 13 \% 13 immatures (Schubart, 1944); Santa Rita [-23.513697º ; -46.414041ºW], 03.xi.1940, ᄋ (Schubart, 1944); Paraná: Curitiba $\left[-25.495342^{\circ} \mathrm{S} ;-49.303308^{\circ} \mathrm{W}\right], 3180^{\circ} 235$ ㅇ (Schubart, 1953); Piraquara [-25.454547º $\mathrm{S}-49.062333^{\circ} \mathrm{W}$ ], Banhado (Schubart, 1953); Santa Catarina: Florianópolis [-27.602630 S; $\left.-48.547169^{\circ} \mathrm{W}\right]$, iv.1946, Gofferjé coll. (Schubart, 1953); Rio Grande do Sul: São Francisco de Paula, Centro de Pesquisas e Conservação da Natureza Pró-Mata [-29.481206º; $\left.-50.173833^{\circ} \mathrm{W}\right], 2016$, P.E.S. Rodrigues et al. coll. (Rodrigues et al., 2017).

\section{Orthomorpha coarctata (Saussure, 1860)} (Figs. 1B, 4A, 5B)

Descriptive notes: See Likhitrakarn et al. (2011: 12, figs. 4-8).

Note: Based on examined material, immatures and females of $O$. coarctata cannot be identified and morphologically distinguished from those of Oxidus gracilis. The species was placed in the genus Asiomorpha by Verhoeff (1939), and its taxonomic position is under discussion (see Likhitrakarn et al., 2011, 2019; Nguyen \& Sierwald, 2013). For a listing purpose, we maintained the species in Orthomorpha according to the latest taxonomic reviews (for more details, see Likhitrakarn et al., 2011, 2019).

Identification: The species can be easily separated from autochthones Neotropical paradoxosomatids mainly by gonopod features (Fig. 5B). Males of $O$. coarctata are recognized by a single terminal lobule on gonopod tip; spikes and denticles either missing or nearly missing (Likhitrakarn et al., 2011, 2019).

Distribution: The species is widely distributed in the tropics (Nguyen \& Sierwald, 2013), occurring in USA, Hawaii (Shelley et al., 1998), Caribbean islands (Nguyen \& Sierwald, 2013), and SE Asia (Korsós, 2004). Orthomorpha coarctata is distributed predominantly in the North and Southeast regions in Brazil, occurring in urban and rural areas, greenhouses, forests, and islands.

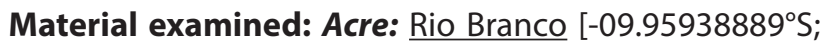
$-67.85665556^{\circ} \mathrm{W}$ ], 09.x.2017, J.A. Rafael coll., (INPA); Amazonas: Boca do Tefé [-03.488378 ${ }^{\circ} \mathrm{S} ;-64.843366^{\circ} \mathrm{W}$ ], mata rio Solimões, ix.1952, Equipe IBSP coll., $60^{\circ} 29$ (IBSP 106); Careiro da Várzea, Ilha do Careiro [-03.16607500 ${ }^{\circ}$; $\left.\quad-59.73265278^{\circ} \mathrm{W}\right], 08 . v i i .1997, \mathrm{~K}$. Vohland coll., $\sigma^{\prime \prime}$ (INPA); Manaus, Residencial Nascentes do Tarumã $\left[-02.99222222^{\circ} \mathrm{S} ; \quad-60.03416667^{\circ} \mathrm{W}\right]$, 05.vii.2016, T. Mahlmann coll., 200 619 (INPA); Igarapé Cururu $\quad\left[-03.12666667^{\circ} \mathrm{S} ; \quad-59.94055556^{\circ} \mathrm{W}\right], \quad 22-24$. ii.2007, N.O. Aguiar et al., coll., + (INPA); Bosque da Ciência [-03.09743889² ; $\left.-59.98781389^{\circ} \mathrm{W}\right], 07 . v .2017$, T.M. Almeida coll., $310^{x} 139$ (INPA); Instituto Nacional de Pesquisas da Amazônia, campus II [-03.096214º'; $-59.989578^{\circ} \mathrm{W}$ ], 20.iv.2016, 20 (INPA); campus INPA, secondary forest $\left[-03.13333333^{\circ} \mathrm{S} ;-60.01666667^{\circ} \mathrm{W}\right]$, 03.viii.1995, J. Adis et al., coll., 21 ㅇ 100' (INPA); campus of Universidade Federal do Amazonas (UFAM) [-03.10024444 $\left.{ }^{\circ} \mathrm{S} ; \quad-59.97850000^{\circ} \mathrm{W}\right], \quad 07 . x .2005, \quad$ M.L. Custódio coll., ơ 2 9 (INPA); 09.ii.2015, N.T.B. Antunes coll., ○ (INPA); Embrapa Amazônia Ocidental, banana plantation, near the Climatology laboratory $\left[-02.893680556^{\circ} \mathrm{S}\right.$; $-59.9730667^{\circ} \mathrm{W}$ ], 28.iv.2016, T.M. Almeida \& A.E.C. Silveira coll., 30 $\$$ (INPA); Reserva Florestal Adolpho Ducke [-02.96334444 $\left.{ }^{\circ} \mathrm{S} ;-59.92283333^{\circ} \mathrm{W}\right], 17 . v .2014$, 70" 6 ㅇ (INPA); 23.vii.2015, 20" (INPA); 20.xii.2018, T.M.

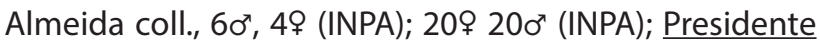
Figueiredo [-01.797656 ${ }^{\circ}$ S; $-59.973303^{\circ} \mathrm{W}$ ], 16.vii.1996, K. Vohland coll., $\$$ ơ $^{\top}$ (INPA); Rondônia: Porto Velho, Parque Municipal $\left[-10.738177^{\circ} \mathrm{S} ;-62.218467^{\circ} \mathrm{W}\right], 02 . i i i .2010$, G. Miranda coll., ơ 2 ㅇ 1 immature (MNRJ); Porto Velho, campus Universidade Federal de Rondônia - UNIR [-08.76349167 $\left.{ }^{\circ} \mathrm{S} ;-63.906575^{\circ} \mathrm{W}\right]$, tree trunk, 14.xi.2016, A. Andriolo coll., o (INPA); Pará: Itaituba [-04.275500S; $-55.992846^{\circ} \mathrm{W}$ ], 08.vii.2003, J. de Fronte coll., 50 3\% (MCTP 124); Belém, Campus MPEG [-01.451628 ${ }^{\circ}$; -48.446535 ${ }^{\circ} \mathrm{W}$ ], 10-30.vi.2010, R. Ott coll., o' 9 (MPEG); Belterra, urban area $\left[-03.161651^{\circ} \mathrm{S}\right.$; $\left.-54.965476^{\circ} \mathrm{W}\right]$, 26-29.x.2009, Equipe IBSP coll., ơ (IBSP 7758); Paraíba: João Pessoa [-07.124538 ${ }^{\circ}$; $\left.-34.845187^{\circ} \mathrm{W}\right]$, O. Schubart 
coll., ơ (MNRJ 11721); x.1935, O. Schubart coll., 16 specimens (MNRJ 11707); 10.x.1935, L. Cordeiro coll., $2 \sigma^{\prime \prime}$ (MNRJ 11692); Pernambuco: Recife [-08.056951ํㅇ; $-34.929493^{\circ} \mathrm{W}$ ], O. Schubart coll., 20 29 (MNRJ 11705); 29 (MNRJ 11690); o" (MNRJ 11687); ㅇ (MNRJ 11712); 0. Schubart coll., 20'3 (MNRJ 11715);29.i.1935, O. Schubart
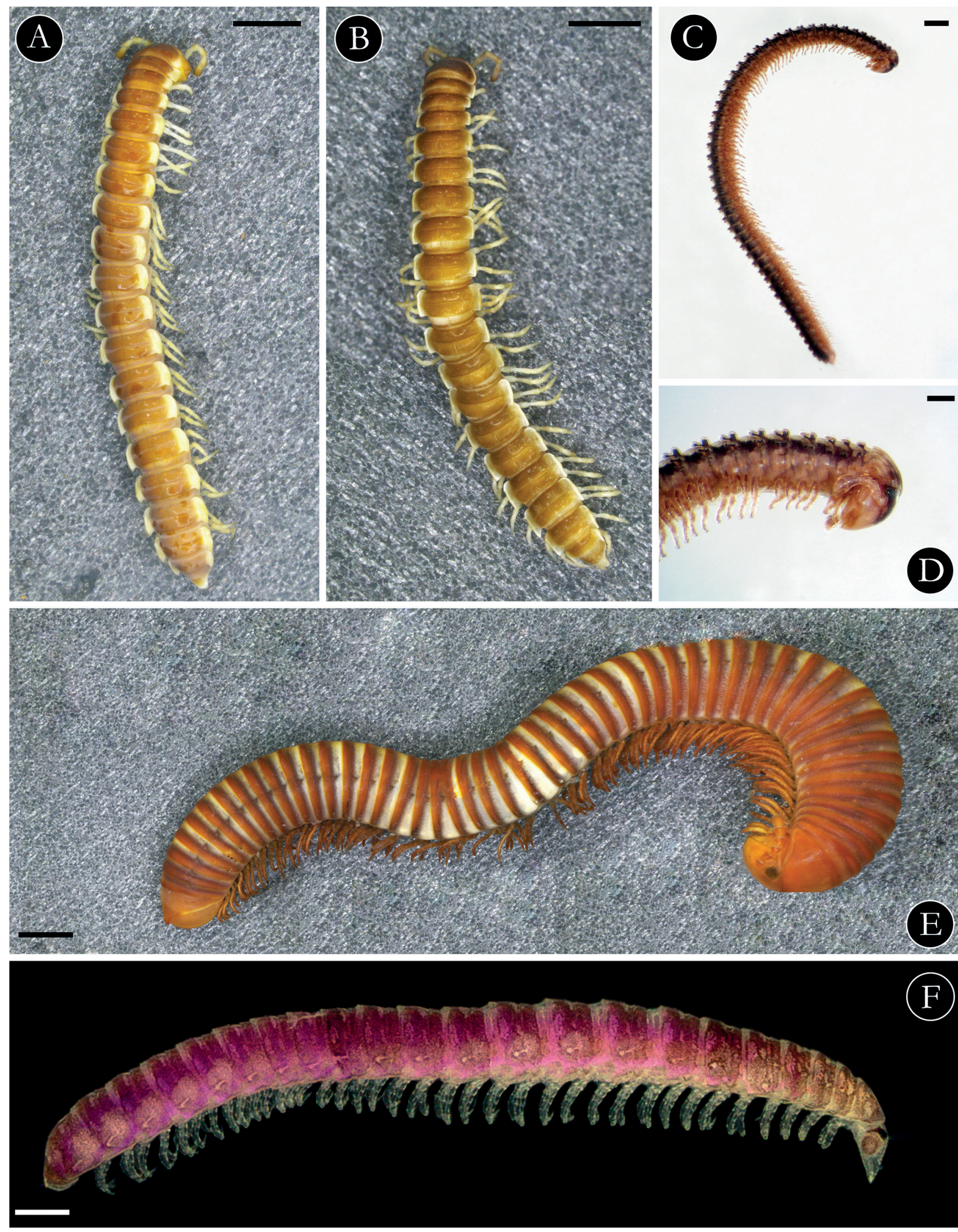

Figure 4. Introduced species in Brazil, habitus: (A) Orthomorpha coarctata; (B) Oxidus gracilis; (C, D) Glyphiulus granulatus; (E) Trigoniulus corallinus; (F) Rhinotus purpureus. Scale bars: $2 \mathrm{~mm}(\mathrm{~A}, \mathrm{~B}, \mathrm{E}) ; 500 \mu \mathrm{m}(\mathrm{C}, \mathrm{D}) ; 200 \mu \mathrm{m}$ (F). 
coll., ơ (MNRJ 11709); Parque Estadual de Dois Irmãos, ix.1936, O. Schubart coll., ơ (MNRJ 11688); Jiquiá, O. Schubart coll., $120^{\top}$ (MNRJ 11699); Tegipió [-08.056961 ${ }^{\circ} \mathrm{S}$; -34.929503W], 06.i.1932, O. Schubart coll., ㅇ 2 immatures (MNRJ 11718); Torres [-08.056958 $\mathrm{S}-34.929500^{\circ} \mathrm{W}$ ], O. Schubart coll., ơ $\$ 1$ immature (MNRJ 11720); Ilha Itamaracá [-08.056961ㅇ; $\left.-34.929503^{\circ} \mathrm{W}\right]$, O. Schubart coll., 240" (MNRJ 11704); Ruínas do Engenho Amparo, O. Schubart coll., ơ 2 ( Noronha [-03.863123ㅇ $\left.-32.440625^{\circ} \mathrm{W}\right]$, Praia do Leão, 10.vi.2019, ơ ๆ (INPA); Ilha Rata, 01-09.vi.2019, J.A. Rafael \& D.M.M. Mendes coll., \& (INPA); Trilha do CapimAçu, 01-09.vi.2019, J.A. Rafael, F. Limeira-de-Oliveira \& D.M.M. Mende coll., 3o' 29 (INPA); Mangue, southeast part of Ilha Rata mangue, 01-09.vi.2019, J.A. Rafael, F. Limeira-de-Oliveira \& D.M.M. Mende coll., o (INPA); Tocantins: Araguaína $\left[-15.200975^{\circ} \mathrm{S} ;-51.852218^{\circ} \mathrm{W}\right]$, São João, 05.xi.2016, K.S. Pacheco coll., $20^{\top} 1$ immature (IBSP 7500); Setor Cimba, 22.x.2014, J. Pereira coll., $0^{7}$ (IBSP 7498); Lageado [-09.871592º ; $-48.297346^{\circ} \mathrm{W}$ ], Área urbana, 23.iv.2002, I. Knysak, R. Martins \& G. Puorto coll., 190' 5 9 6 immatures (IBSP 1556); Palmas $\left[-09.760156^{\circ} \mathrm{S} ;-48.378491^{\circ} \mathrm{W}\right]$, U.H.E. Luís Eduardo Magalhães, 22.xi.2000, I. Knysak, R. Martins \& G. Puorto coll., ơ (IBSP 857); 22.xi.2000, R. Martins \& G. Puorto coll., $80^{7} 1491$ immature (IBSP 846); 21.iii.2001, R. Martins \& G. Puorto coll., o' 2 ㅇ 1 immature (IBSP 880); $\sigma^{7}$ \% (IBSP 881); ९ (IBSP 873); ᄋ (IBSP 863); 40 4 1 immature (IBSP 883); Porto Nacional $\left[-10.759459^{\circ} \mathrm{S} ;-48.398068^{\circ} \mathrm{W}\right.$ ], Ribeirão Santa Luzia, U.H.E. Luís Eduardo Magalhães, 20.i.2000, I. Knysak, R. Martins \& G. Puorto coll., 40" ㅇ (IBSP 938); Wanderlândia [-06.929806 ${ }^{\circ}$; $-48.006315^{\circ} \mathrm{W}$ ], 30.ix.2014, o' (IBSP 7499). Mato Grosso: Cuiabá [-15.600776%; $-56.074270^{\circ} \mathrm{W}$ ], 14.v.2015, Francisco coll., 30' (UFMT); Pedregal, 12.vii.2012, R. Pinto coll., o' (UFMT); campus UFMT, 17.viii.2016, B. Martins coll., ơ (UFMT); 23.v.2017, T. Amorim coll., 20'3 (UFMT); 26.vi.2017, G. Brunna coll., $\sigma^{7}$ (UFMT); xii.2012, V.S. Falcio coll., ơ (UFMT); 09.vii.2017, J.R. Silva coll., 2o' (UFMT); 19.viii.2016, K. Fonseca \& M. Martello coll., $2 \sigma^{\prime}$ \% (UFMT); CPA III, 17.ii.2011, C.C.L. Dias coll., ơ (UFMT); 15.v.2011, A.F.S. Assis coll., o" (UFMT); Bairro Tijucal, 18.vii.2010, R. Moraes coll., o (UFMT); Várzea Grande [-15.650197 S; -56.132670W], 16.ix.2013, A.C. Santos coll., ơ (UFMT); Minas Gerais: Belo Horizonte

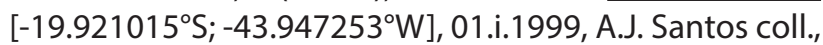
$\sigma^{\top 1}$ P (IBSP 1306); FAE UFM [ $\left[-19.873236^{\circ} \mathrm{S} ;-43.966837^{\circ} \mathrm{W}\right]$, iv.2006, L. Bernardi coll., 40" $\$$ (IBSP 2910); Raul Soares, São Vicente da Estrela [-19.960899 ${ }^{\circ}$; $-42.438840^{\circ} \mathrm{W}$ ], 06.i.2002, E.N. de Jesus coll., o' 2 \% (IBSP 961); Cordisburgo [-19.067019 ${ }^{\circ}$; $\left.-44.215763^{\circ} \mathrm{W}\right]$, 25.x.1947, O. Schubart

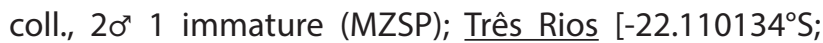
$-43.208401^{\circ} \mathrm{W}$ ], Road to Rio de Janeiro, 12.x.1947, O. Schubart coll., $50^{\prime}$ ㅇ 2 immatures (MZSP); $20^{\prime \prime} 793$ immatures (MZSP); Espírito Santo: Sooretama [-19.178587º ; $-40.098118^{\circ} \mathrm{W}$ ], plantação de café, 24.ii.2011, A.F.R. Teixeira coll., $\sigma^{\pi}$ \& (MCN 1184); $\sigma^{\pi}$ ○ (MCN 1218); 우 (MCN 1183); o" (MCN 1185); 20" (MCN 1210); $5 \sigma^{\prime \prime} 8$ \% (MCN 1199); 407 7\% (MCN 1188); 30.iii.2011, A.F.R. Teixeira coll., 270" 53 \% 18 immature (MCN); 24.iv.2011, A.F.R. Teixeira coll., $2 \sigma^{\circ} 391$ immature (MCN); 25.v.2011, A.F.R.
Teixeira coll., ơ (MCN); Rio de Janeiro: Mangaratiba, Rio Junqueira $\left[-22.930954^{\circ} \mathrm{S} ; \quad-44.038933^{\circ} \mathrm{W}\right], \quad \mathrm{O}$. Schubart coll., 719 (MNRJ 11697); Rio de Janeiro [-22.921765 ${ }^{\circ}$; -43.169510 W], 28.iii.1951, J. Becker coll., $20^{\pi}$ ○ (MNRJ); O. Schubart coll., ơ (MZSP); São Paulo: Ubatuba [-23.446317º ; -45.087149 $\mathrm{W}$ ], 12-13.ix.1998,

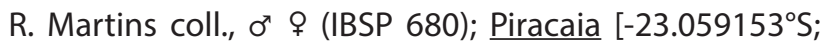
$\left.-46.360347^{\circ} \mathrm{W}\right], x .1996, \mathrm{~S}$. Rocha coll., o' (IBSP 691); Itu [-23.272062 $\left.{ }^{\circ} ;-47.299290^{\circ} \mathrm{W}\right], 11 . i v .1985$, R. D'Ávila coll., $130^{\prime} 4$ 오 (IBSP 667); São Paulo, Jardim Rizzo [-23.572008º'; $-46.732739^{\circ} \mathrm{W}$ ], 20.xi.1998, Eq. IBSP coll., $0^{\top 7}$ (IBSP 7759); Paraná: Curitiba $\left[-25.495342^{\circ} \mathrm{S} ;-49.303308^{\circ} \mathrm{W}\right], 20^{\prime \prime}$ ㅇ (IBSP 7760).

Historical records: Amazonas: Manaus [-03.023045웅 -59.965390W], Bicego coll. (Brölemann, 1904); Embrapa Amazônia Ocidental $\left[-02.893744^{\circ} \mathrm{S} ; \quad-59.973109^{\circ} \mathrm{W}\right]$ (Hofffman et al., 2002); Paraíba: João Pessoa [-07.11505; -34.8631 $\mathrm{W}$ ], 10.vii.1937, E. Cordeiro coll. (Schubart, 1939); Pernambuco: Recife [-08.0539² $\mathrm{S}-34.8811^{\circ} \mathrm{W}$ ], Bairro dos Afogados, 1935 (Schubart, 1942a); margin of Rio Capibaribe, 30.i.1935 (Schubart, 1942a); margin of Rio Beberibe, 31.iii.1935 (Schubart, 1942a); Madalena, 25.iv.1935 (Schubart, 1942a); Bairro Torre, 16.vii.1936 (Schubart, 1942a); Bairro Dois Irmãos, 1934 (Schubart, 1939); Bairro Tegipio, 1934 (Schubart, 1939); Bairro Várzea, 1934 (Schubart, 1939); Olinda [-08.0089% ; $-34.8553^{\circ} \mathrm{W}$ ], banana plantation, 07.iv.1935 (Schubart, 1939); Iguarassú $\left[-07.8333^{\circ} \mathrm{S} ;-34.9000^{\circ} \mathrm{W}\right]$, Ilha Itamaracá $\left[-07.754660^{\circ} \mathrm{S}\right.$; -34.837309 W], 25.v.1935 (Schubart, 1939); São Lourenço da Mata, 14.vii.1937,(Schubar, 1939); Alagoas: Jequiá da Praia [-09.7811 $\left.{ }^{\circ} \mathrm{S} ;-36.0936^{\circ} \mathrm{W}\right]$, Lagoa de Jequiá, Porta da Boca, 10.vii.1936, ㅇ 1 immature (Schubart, 1939); Goiás: Aragarças $\left[-15.912823^{\circ} \mathrm{S} ;-52.251231^{\circ} \mathrm{W}\right], \mathrm{x} .1953$, H. Sick coll., ơ (Schubart, 1958a); Rio de Janeiro: Rio de Janeiro, Bairro Cachambí [-22.902038º'; $-43.273901^{\circ} \mathrm{W}$ ] (Schubart, 1945b); Bairro Deodoro [-22.857562 ${ }^{\circ}$; $-43.384850^{\circ} \mathrm{W}$ ] (Schubart, 1945b); Bairro do Encantado [-22.896400 ${ }^{\circ}$; $\left.-43.302050^{\circ} \mathrm{W}\right]$ (Schubart, 1945b); Bairro Jacarepaguá [-22.971732 ${ }^{\circ} \mathrm{S} ;-43.391675^{\circ} \mathrm{W}$ ] (Schubart, 1945b); Lagoa Rodrigo de Freitas [-22.973385' $-43.207108^{\circ} \mathrm{W}$ ] (Schubart, 1945b); Bairro do Leblon [-22.984645 ${ }^{\circ}$; $-43.223162^{\circ} \mathrm{W}$ ] (Schubart, 1945b); Morro dos Dois Irmãos [-22.952544 S; -43.399345W] (Schubart, 1945b); Serra de Bangú [-22.876050 $\mathrm{S}$; $-43.468651^{\circ} \mathrm{W}$ ] (Schubart, 1945b); Bairro Tijuca $\left[-22.935505^{\circ} \mathrm{S}\right.$; $-43.243112^{\circ} \mathrm{W}$ ] (Schubart, 1945b); São Gonçalo, Engenho Novo [-22.903896 ${ }^{\circ}$; $-43.268490^{\circ} \mathrm{W}$ ] (Schubart, 1945b); São Paulo: Pirassununga [-22.067267 ${ }^{\circ} \mathrm{S} ;-47.395011^{\circ} \mathrm{W}$ ], 17.i.1940, ơ 91 immature (Schubart, 1944); 03.iv.1940, 90' 69 (Schubart, 1944); 22.v.1940, 80' 3ㅇ 1 immature (Schubart, 1944); 23.vii.1940, 30 7o 12 immatures (Schubart, 1944); 13.ii.1941, 40 29 (Schubart, 1944); 02.iii.1940, 30" 2910 immatures (Schubart, 1944); 26.ix.1941, 7o 4ㅇ 2 immatures (Schubart, 1944); 12.iii.1940 (Schubart, 1944); 27.ii.1940, A. Aguirre coll., $110^{7} 11914$ immatures (Schubart, 1944); Fazenda Pedra Branca, 11.i.1942, J. Gaspar coll., ㅇ 2 immatures (Schubart, 1944); Fazenda São Domingos, 22.ix.1940, $200^{\top 1} 14$ ? (Schubart, 1944). 


\section{Family Haplodesmidae}

\section{Prosopodesmus jacobsoni Silvestri, 1910} (Figs. 1C, 6A)

Descriptive notes: See Brölemann (1920: 226, figs. 141-144).

Identification: Prosopodesmus jacobsoni can be separated from other species of Neotropical Haplodesmidae by having mushroom-shaped porosteles (see Enghoff, 1993: fig. 4); telopodite with large lateral lamella; two subapical dentiform processes (Silvestri, 1910: fig. 7; Fig. 11a; Mesibov, 2012: fig. 4).

Distribution: The species is widespread in the tropics and supposedly introduced by human activities (Hoffman, 1999). Some records have been made from USA and Hawaii, West Indies, Cape Verde, Tanzania, India, Indonesia, Fiji, and Taiwan (Loomis, 1950; Enghoff, 1993; Hoffman, 1999; Shelley \& Golovatch, 2000; Akkari \& Enghoff, 2011; Golovatch et al., 2011; Mesibov, 2012). Its native distribution is uncertain (Mesibov, 2012). In Brazil, the species has been recorded only in urban areas from states of Rio de Janeiro and São Paulo. All records were obtained from the literature.

Historical records: Rio de Janeiro: Niterói, Icaraí [-22.903431 $\left.{ }^{\circ} \mathrm{S} ;-43.111570^{\circ} \mathrm{W}\right]$ (Schubart, 1947); Rio de Janeiro [-22.921765 ${ }^{\circ}$; $\left.-43.169510^{\circ} \mathrm{W}\right]$ (Schubart, 1947); Bairro Tijuca $\left[-22.935505^{\circ} \mathrm{S} ;-43.243112^{\circ} \mathrm{W}\right]$, 17.v.1938, O. Schubart coll., 30 $30^{7} 1$ immature (Schubart, 1945b); 08.x.1938, o" (Schubart, 1945b); 29.x.1938, O. Schubart coll., 20 (Schubart, 1945b); 04.xi.1939, O. Schubart coll., 우 (Schubart, 1945b); 06.xi.1939, O. Schubart coll., o' 우 (Schubart, 1945b); 08.xi.1939, O. Schubart coll., 30" ? 1 immature (Schubart, 1945b); 09.xi.1939, O. Schubart coll., 20" 2o (Schubart, 1945b); 12.xi.1939, O. Schubart coll., o" (Schubart, 1945b); 13.xi.1939, O. Schubart coll., ơ (Schubart, 1945b); 27.xi.1939, O. Schubart coll., 20" (Schubart, 1945b);05.vi.1940, O. Schubart coll., o' (Schubart, 1945b); 23.ix.1939, O. Schubart coll., o (Schubart, 1945b); São Paulo: Mogi Guaçu [-22.185269º ; $-47.097326^{\circ} \mathrm{W}$ ] (Schubart, 1947); Pirassununga $\left[-22.067267^{\circ} \mathrm{S}\right.$; -47.395011W], Fazenda São Domingos (Schubart, 1947).

\section{Order Spirostreptida \\ Suborder Cambalidea \\ Family Cambalopsidae}

\section{Trachyjulus calvus (Pocock, 1893a)} (Figs. 1D, 6C-D)

Descriptive notes: Male, see Golovatch et al. (2012: 114, figs. 8-9).

Identification: T. calvus is easily separated from Brazilian species of Spirostreptida by having tergites longitudinally crested (Golovatch et al., 2012: fig. 8) and the presence of posterior gonopod. According to the diagnosis made by Golovatch et al. (2012), males are recognized by lateral coxal process of the anterior gonopod being very slender (Fig. 6C); slender and conical medial coxal process; posterior gonopod with axe-shaped flagellum extended and with microgranulate distal lobules (Fig. 6D).

Distribution: T. calvus presents a tropical distribution (Golovatch et al., 2012). In Brazil, the species has been recorded only in urban areas from states of Rio de Janeiro and Bahia (ca. 1,500 km).

Material examined: Bahia: Salvador, campus UFBA [-13.004364º ; $\left.-38.508978^{\circ} \mathrm{W}\right], 2013$, C.M.P. Leite coll., o' (UFMT).

Records: Rio de Janeiro: Rio de Janeiro, Bairro Tijuca [-22.935505 ${ }^{\circ}$; $\left.-43.243112^{\circ} \mathrm{W}\right], 17 . v .1938$, A.C. Aguirre coll., 11 ㅇ 1 immature (Schubart, 1946b); vi.1938, A.C. Aguirre coll., + (Schubart, 1946b);21.v.1939, A.C. Aguirre coll., 30’ 9 ㅇ 8 immatures (Schubart, 1946b); 12.ix.1939, A.C. Aguirre coll., ơ 1 immature (Schubart, 1946b); 29.x.1939, A.C. Aguirre coll., 6 immatures (Schubart, 1946b); 27.xi.1939, A.C. Aguirre coll., 4 immatures (Schubart, 1946b); 30.i.1940, A.C. Aguirre coll., 30 49 (Schubart, 1946b); 30.iv.1940, A.C. Aguirre coll., 594 immatures (Schubart, 1946b); 05.vi.1940, A.C. Aguirre coll., ㅇ (Schubart, 1946b); 11.vi.1940, A.C. Aguirre coll., 4 ㅇ 3 immatures (Schubart, 1946b); 04.viii.1940, A.C. Aguirre coll., $20^{\prime} 4$ ㅇ 2 immatures (Schubart, 1946b); 14.ix.1940, A.C. Aguirre coll., 507 109 1 immature (Schubart, 1946b); 30.vii.1939, A.C. Aguirre coll., ㅇ (Schubart, 1946b); 15.ix.1939, A.C. Aguirre coll., 930" 133927 immatures (Schubart, 1946b); 13.iv.1946, A.C. Aguirre coll., 20 4 ㅇ 4 immatures (Schubart, 1946b).

\section{Glyphiulus granulatus (Gervais, 1847) (Figs. 1C, 4C-D, 6B)}

Descriptive notes: See Golovatch et al. (2007: 12, figs. 1-6).

Identification: Males of G. granulatus are recognized by a median outgrowth of the coxosternum in the anterior gonopods (Golovatch et al., 2007: fig. 5a, b; Fig. 6B) and the typical pattern of carinotaxy. As in T. calvus, the species is easily identified when compared with Brazilian species of Spirostreptida by having tergites longitudinally crested (Fig. 4C-D) and the presence of posterior gonopod (see Golovatch et al., 2007: 12).

Distribution: G. granulatus has been recorded in SE Asia and in islands in the Pacific and Indian oceans (Shelley, 1998a; Jeekel, 2004; Korsós, 2004; Enghoff et al., 2015). In Brazil, the species occurs only in urban areas in Santa Maria, state of Rio Grande do Sul.

Material examined: Rio Grande do Sul: Santa Maria [-29.6914 ${ }^{\circ}$; $\left.-53.8008^{\circ} \mathrm{W}\right]$, Universidade Federal de Santa Maria, 17.iv.2014, V.M. Silva coll., $80^{\top} 5$ (MCN). 

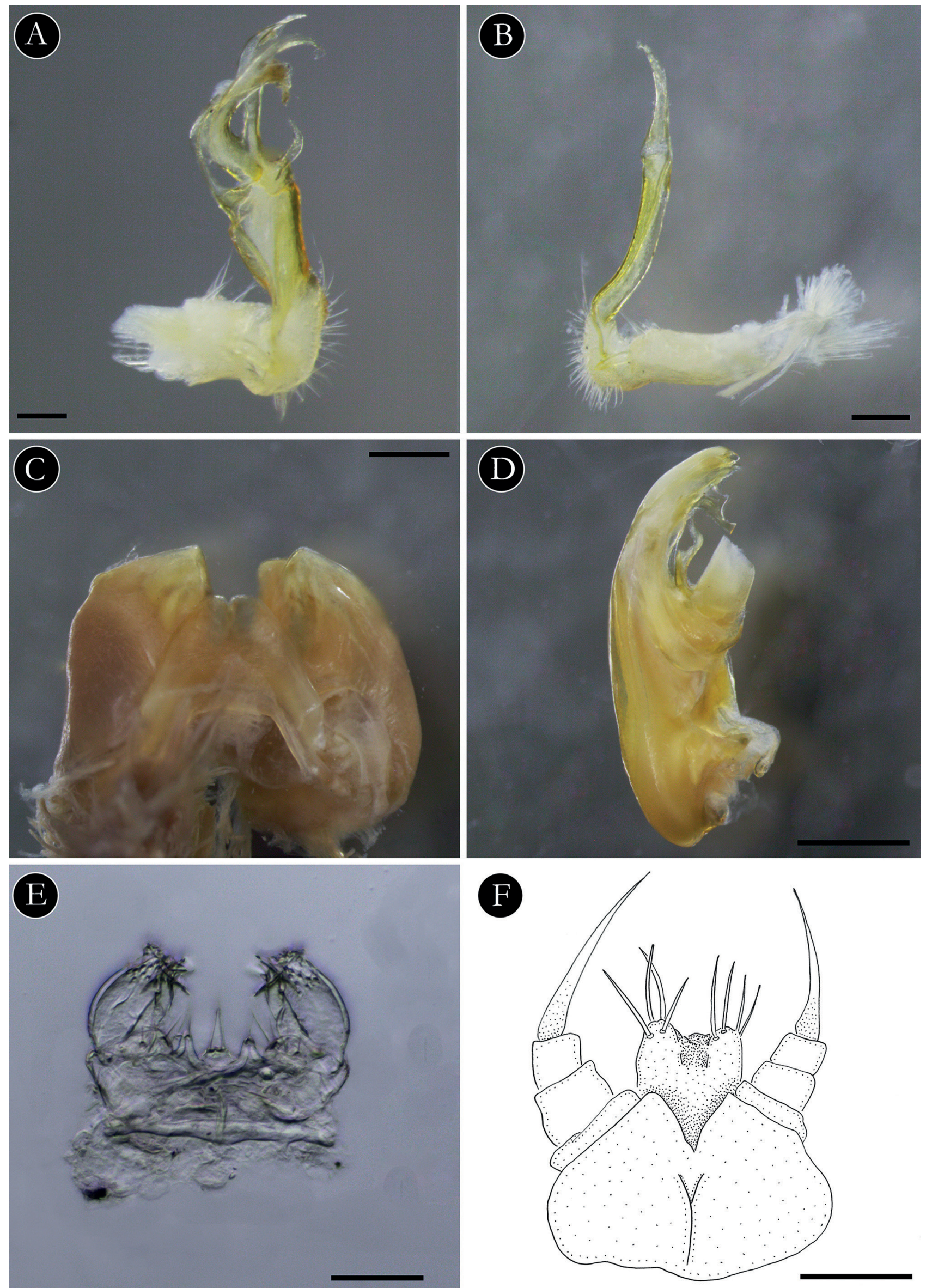

Figure 5. (A) Oxidus gracilis, gonopod in mesal view; (B) Orthomorpha coarctata, gonopod in mesal view; (C) Trigoniulus corallinus, anterior gonopods in anterior view; (D) Trigoniulus corallinus, telopodite of left posterior gonopod in anterior view; (E) Rhinotus purpureus, anterior gonopod in posterior view; (F) Rhinotus purpureus, posterior gonopod in posterior view. Scale bars: $100 \mu \mathrm{m}$ (A, B, E, F); $750 \mu \mathrm{m}$ (C); 1 mm (D). 


\section{Order Spirobolida \\ Family Pachybolidae}

\section{Trigoniulus corallinus (Gervais, 1842)}

(Figs. 2A, 4E, 5C-D)

Descriptive notes: See Shelley \& Lehtinen (1999: 1389, figs. 8-14).

Note: An overview of its taxonomic status was made by Shelley \& Lehtinen (1999).

Identification: The species is easily recognized by the strongly reddish color in life (Fig. 9e). According to the diagnosis made by Shelley \& Lehtinen (1999, figs. 11-13), males of $T$. corallinus are recognized by the coxae of the anterior gonopods narrowly separated by subtriangular sternum indented in midline; telopodites subtriangular, extending directly mediad (Fig. 5C), telopodites of the posterior gonopods rounded and with broad medial lobe at midlength; two inner projections arising basally from lobe and directed distad; and outer projection with notch on inner margin, expanded distad (Fig. 5D).

Distribution: Trigoniulus corallinus presents a tropical distribution (Hoffman, 1999; Shelley, 1998b; Shelley \& Lehtinen, 1999; Korsós, 2004; Enghoff et al., 2015). In Brazil, the species is distributed predominantly in the North and Northeast regions, occurring in urban and rural areas, greenhouses, and forests. Records from the state of Rio de Janeiro are due to its use for the production of organic compost for agriculture (Antunes et al., 2016, 2019). Species of Trigoniulinae distributed in the Neotropical region are supposedly introduced from SE Asia (see Hoffman, 1994; Hoffman et al., 1996).

Material examined: Roraima: Caracaraí, Serra da Mocidade $\quad\left[01.491084^{\circ} \mathrm{S} ; \quad-61.78333333^{\circ} \mathrm{W}\right], \quad 25 . \mathrm{i}-06$. ii.2016, M. Oliveira, F.F. Xavier \& T. Mahlmann coll., $\sigma^{7}$ (INPA); Parque Nacional Serra da Mocidade, $\left[01.074634^{\circ} \mathrm{S} ; \quad-61.900000001^{\circ} \mathrm{W}\right], \quad 15-26 . i .2016$, F.F. Xavier, R. Boldrini \& P. Barroso coll., $20^{\circ} 29$ (INPA); Igarapé Caicubi $\left[00.560220^{\circ} \mathrm{S} ;-62.168280^{\circ} \mathrm{W}\right]$, Pupunha, pitfall, 22.x.2008, Ana coll., o $\$$ (INPA); Amazonas: Manaus, campus INPA [-03.0960583 ${ }^{\circ}$; $-59.9894389^{\circ} \mathrm{W}$ ], 12.vii.2016, D.P. Cordeiro coll., ơ (INPA); Pará: Belém, Bairro Terra Firme [-01.457070 ${ }^{\circ}$; $\left.-48.451254^{\circ} \mathrm{W}\right], 22-23$. vi.2010, R. Ott coll., 40 69 (MCN 659); 17o 11 \% 3 immatures (MPEG Myr-00048); Tocantins: Araguaína [-15.200975 ${ }^{\circ}$; $\left.-51.852218^{\circ} \mathrm{W}\right]$, campus UFT, 02.xi.2016, M. Lopes coll., $0^{7}+$ (IBSP 7494); 13.xi.2016, M. Lopes coll., Quintal de Casa, 4o 39 (IBSP 7495); Bairro da Cimba, 08.viii.2015, F. Costa coll., ơ $q$ (IBSP 7496); 16.ix.2014, V.S. Marinho coll., Urban area, $ᄋ$ (IBSP 7497); Pernambuco: Recife $\left[-08.056951^{\circ} \mathrm{S} ;-34.929493^{\circ} \mathrm{W}\right], 26 . v i .1946$, M.L. Siqueira coll., $20^{7}$ (MZSP); campus Instituto Ricardo Brennand [-08.055537 ${ }^{\circ}$; $-34.959112^{\circ} \mathrm{W}$ ], 27.viii.2010, R. Ott coll., ơ 8 ( $(\mathrm{MCN} 611) ;$ Rio de Janeiro: Rio de Janeiro [-22.921765º; -43.169510W], 13.xii.1954, H. Lopes coll., $0^{7}$ (MZSP).

Historical records: Amazonas: Manaus $\left[-03.023045^{\circ} \mathrm{S}\right.$;

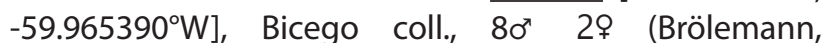
1902); Embrapa Amazônia Ocidental $\left[-02.893744^{\circ} \mathrm{S}\right.$; -59.973109 W] (Hofffman et al., 2002); Pará: Ananindeua, Aurá [-01.408310 $\left.{ }^{\circ} ;-48.397542^{\circ} \mathrm{W}\right], 05 . i i i .1958$, L.Travassos coll., 30 10 (Schubart, 1958b); 04.iii.1958, \& (Schubart, 1958b); Entrada de Utinga [-01.42599 ${ }^{\circ} \mathrm{S} ;-48.444631^{\circ} \mathrm{W}$ ], 12.iii.1958, L. Travassos coll., $20^{\top} 2$ (Schubart, 1958b); Pernambuco: Recife $\left[-08.0539^{\circ} \mathrm{S} ;-34.8811^{\circ} \mathrm{W}\right]$, Bairro dos Afogados, 14.xiii.1934, O. Schubart coll. (Schubart, 1958b); Bairro do Payssandú, 26.iv.1946, M.L. Siqueira coll., 40 6929 immature (Schubart, 1958b); Rio de Janeiro: Rio de Janeiro, Bairro Leblon $\left[-22.985714^{\circ} \mathrm{S}\right.$; $\left.-43.222412^{\circ} \mathrm{W}\right]$, 25.xii.1941, A.C. Aguirre coll., $20^{\circ} 49$ (Schubart, 1958b); Bairro Brás de Pina $\left[-22.831870^{\circ} \mathrm{S}\right.$; $\left.-43.296731^{\circ} \mathrm{W}\right]$, 25.iii.1947, A.C. Aguirre coll., $20^{\circ} 0^{\prime \prime}$ immature (Schubart, 1958b); Bairro do Andaraí [-22.927367\% ${ }^{\circ}$; $-43.251521^{\circ} \mathrm{W}$ ], i.1953, J. Becker coll., 29 (Schubart, 1958b); campus Universidade Rural, km 47 [-22.768546 ${ }^{\circ} \mathrm{S}$; $-43.687338^{\circ}$ W], xii.1957, H.S. Lópes coll., ơ 29 (Schubart, 1958b); Seropédica [-22.768582 ${ }^{\circ}$; $\left.-43.706134^{\circ} \mathrm{W}\right], 2017$, L.F.S. Antunes et al. coll. (Antunes et al., 2019).

\section{Leptogoniulus sorornus (Butler, 1876) (Figs. 2B, 6E-F)}

Descriptive notes: See Shelley \& Lehtinen (1999: 1383, figs. 1-7).

Note: An overview of its taxonomic status was made by Shelley \& Lehtinen (1999).

Identification: According to the diagnosis made by Shelley \& Lehtinen (1999), males of $L$. sorornus are recognized by coxae of the anterior gonopods widely separated by apically broad and subtruncate sternum (Fig. 6E), and telopodites apically uncinated. Telopodites of the posterior gonopods with broad medial lobe, and apically rounded with notches along the distal margin (Fig. 6F).

Distribution: Leptogoniulus sorornus presents a tropical distribution (Hoffman, 1999; Shelley \& Lehtinen, 1999; Korsós, 2004). In Brazil, the species has been recorded in the old-named state of Guanabara (now known as Rio de Janeiro), São Mateus, and Salvador (approximately $1,500 \mathrm{~km}$ from Rio de Janeiro) (Shelley \& Lehtinen, 1999). All records were obtained from the literature.

Historical records: Bahia: Salvador $\left[-12.9711^{\circ} \mathrm{S}\right.$; $\left.-38.5108^{\circ} \mathrm{W}\right]$, J. Becker coll. (Schubart, 1958b); Espírito Santo: São Mateus [-18.718937 ${ }^{\circ}$; $\left.\quad-39.861257^{\circ} \mathrm{W}\right]$ (Schubart, 1947); Rio de Janeiro: Rio de Janeiro [-22.9028 ${ }^{\circ}$; $\left.-43.2075^{\circ} \mathrm{W}\right]$ (Schubart, 1958b); Corcovado, Jardim Botânico, Caullery coll. (Brölemann, 1929); São Gonçalo [-22.8269 ${ }^{\circ}$; $\left.-43.0539^{\circ} \mathrm{W}\right]$, Fazenda Engenho Novo, A.C. Aguirre coll. (Schubart, 1947). 
(4)

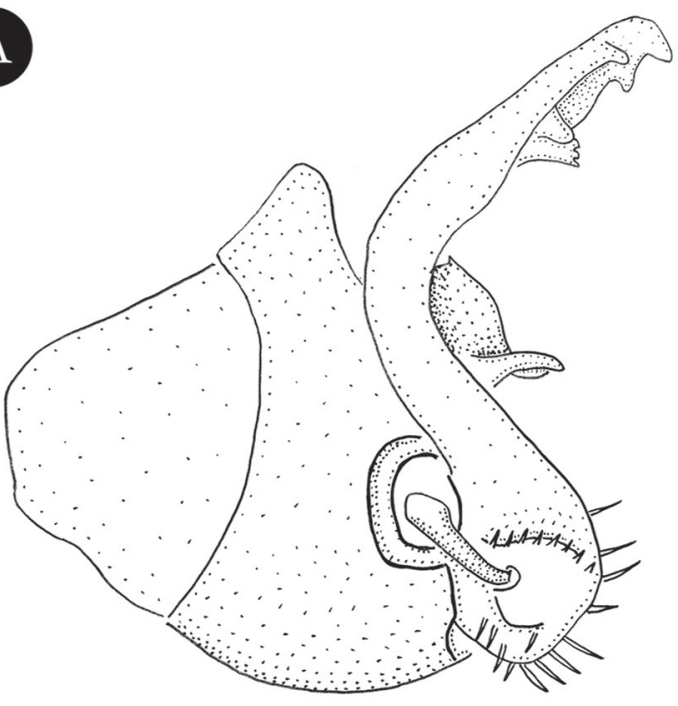

C

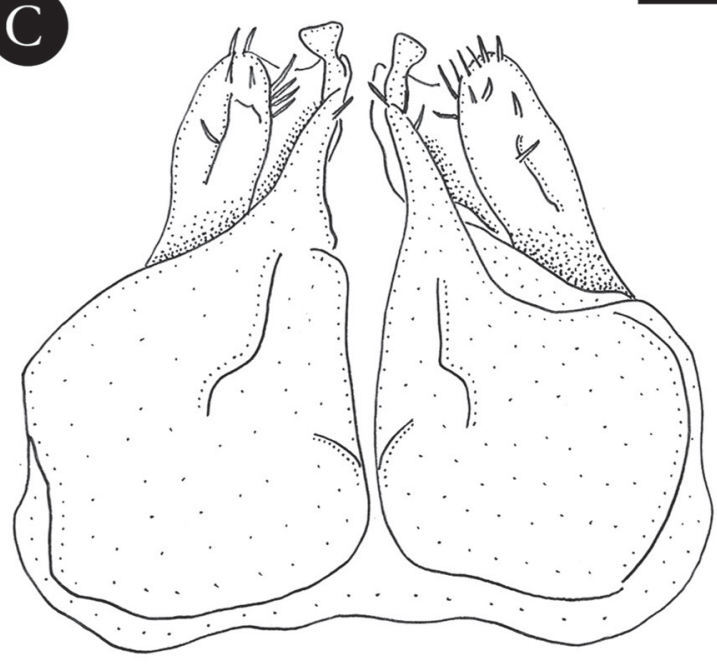

E

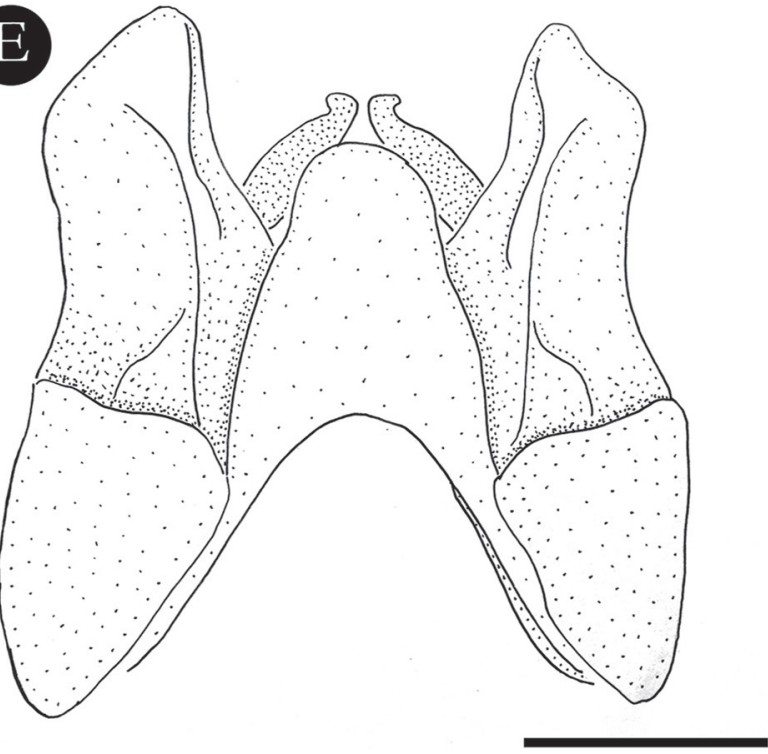

B

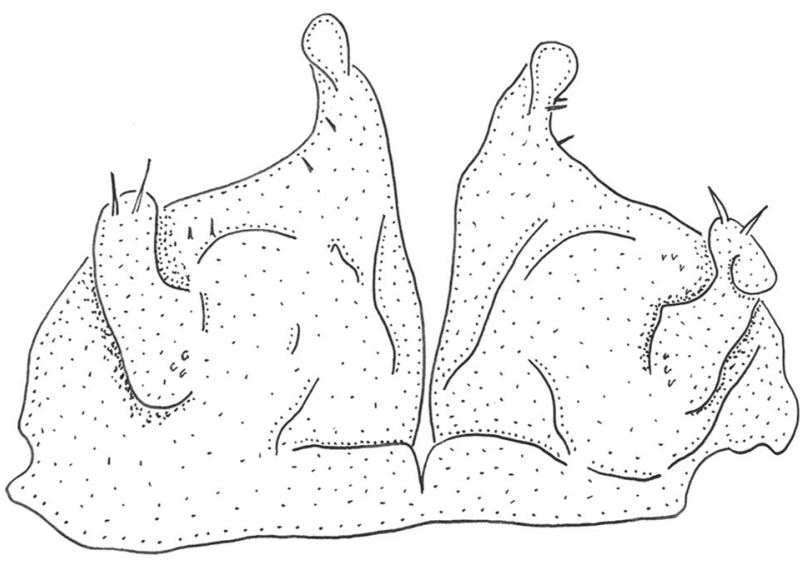

D

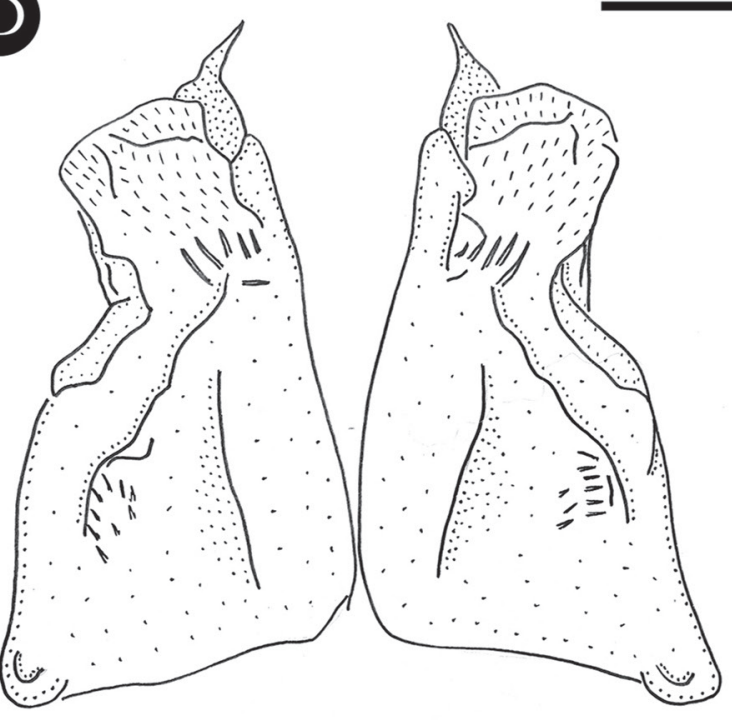

F

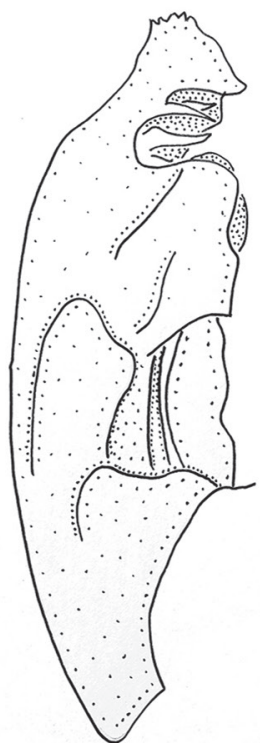

Figure 6. (A) Prosopodesmus jacobsoni, gonopod in mesal view (after Silvestri, 1910); (B) Glyphiulus granulatus, anterior gonopods in posterior view; (C) Trachyjulus calvus, anterior gonopods in anterior view; (D) Trachyjulus calvus, posterior gonopods in anterior view (after Schubart, 1946); (E) Leptogoniulus sorornus, anterior gonopods in anterior view; (F) Leptogoniulus sorornus, telopodite of left posterior gonopod in anterior view (after Shelley \& Lehtinen, 1999). Scale bars: $20 \mu \mathrm{m}$ (B); $100 \mu \mathrm{m}$ (C, D); $750 \mu \mathrm{m}$ (E); 1 mm (F). Image (A) not to scale. 

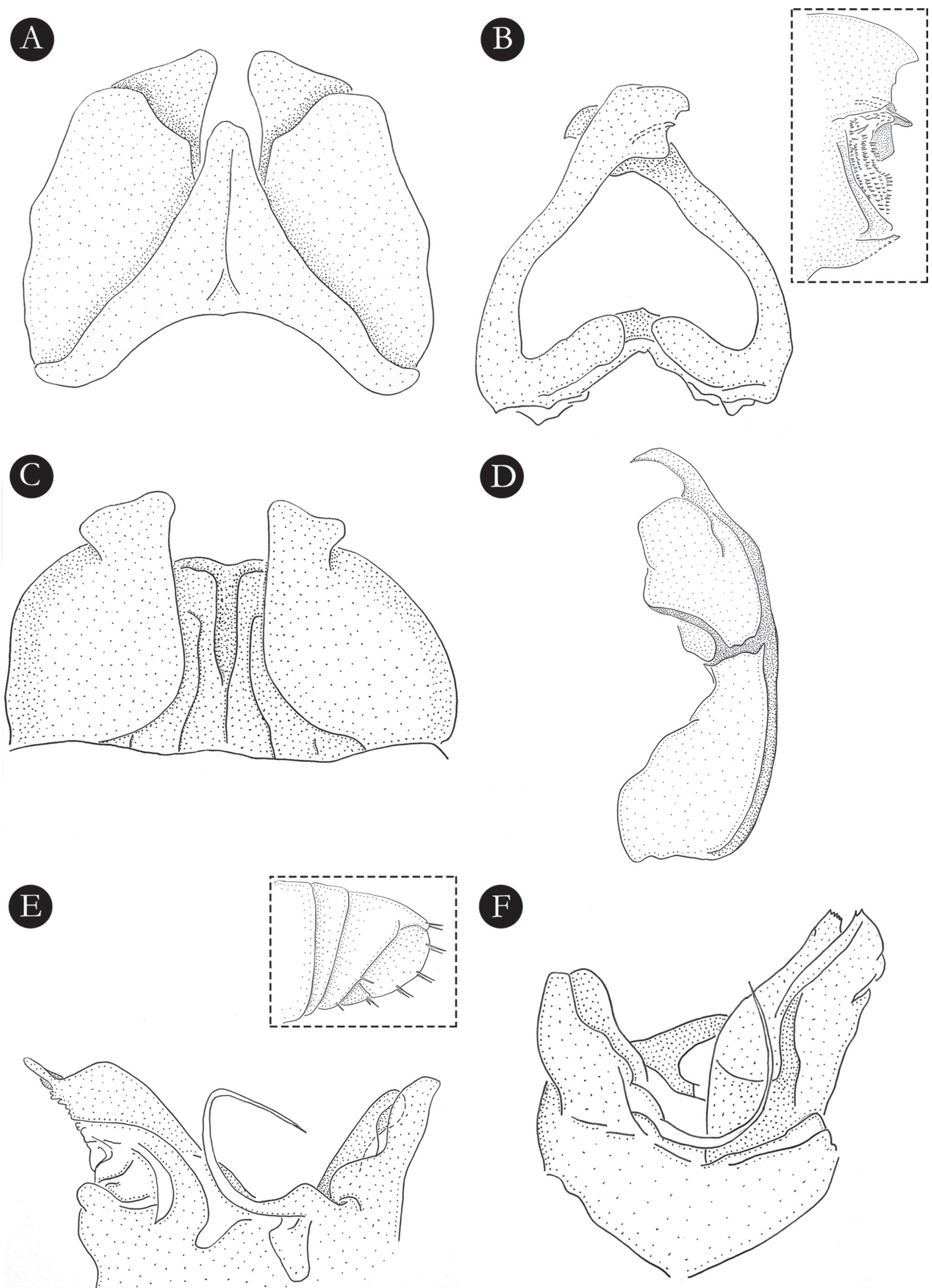

Figure 7. (A) Epitrigoniulus cruentatus, anterior gonopods in posterior view (after Brölemann, 1903); (B) Epitrigoniulus cruentatus, posterior gonopods in anterior view (after Brölemann, 1903). Distal region in detail; (C) Paraspirobolus lucifugus, anterior gonopods in anterior view; (D) Paraspirobolus lucifugus, posterior gonopod in anterior view (after Attems, 1900); (E) Cylindroiulus britannicus, gonopod in anterior view. Detail of telson in lateral view (after Blower, 1985); (F) Cylindroiulus truncorum, gonopod in anterior view (after Blower, 1985). Images not to scale. 


\section{Epitrigoniulus cruentatus (Brölemann, 1903)} (Figs. 1D, 7A-B)

Descriptive notes: See Brölemann (1903: 250, figs. 5-9).

Identification: According to the description made by Brölemann (1903), males of E. cruentatus can be recognized by coxae of the anterior gonopods separated by subtriangular sternum (Fig. 7A); and telopodites subtriangular (Brölemann, 1903: fig. 5). Telopodites of the posterior gonopods rounded and expanded distad; and with notches along the mesal margin (Brölemann, 1903: figs. 6-8; Fig. 7B).

Distribution: Epitrigoniulus cruentatus occurs in the Indo-Malayan region (Brölemann, 1903; Schubart, 1947). In Brazil, the species has been recorded only in the Amazonian region (Brölemann, 1903, 1909).

Historical records: Amazonas: Manaus [-03.063877 ${ }^{\circ} \mathrm{S}$; $-60.036493^{\circ} \mathrm{W}$ Embrapa Amazônia Ocidental [-02.893744 ${ }^{\circ} \mathrm{S}$; $\left.-59.973109^{\circ} \mathrm{W}\right]$ (Hofffman et al., 2002); Pará: Belém [-01.451628 ${ }^{\circ}$; $-48.446535^{\circ} \mathrm{W}$ ] (Schubart, 1947).

\section{Family Spirobolellidae}

\section{Paraspirobolus lucifugus (Gervais, 1837) (Figs. 2C, 7C-D)}

Descriptive notes: See Attems (1900: figs. 13-16) and Brölemann (1902: 184, figs. 223-227).

Identification: According to the descriptions made by Attems (1900) and Brölemann (1902), males of P. lucifugus can be recognized by the anterior and posterior gonopods. Coxae of the anterior gonopods separated by wide sternum slightly indented in midline (Attems, 1900: figs. 13-14; Brölemann, 1902: fig. 226; Fig. 7C). Telopodites of the posterior gonopods apically fusiform and with a broad lobe (Attems, 1900: fig. 16; Fig. 7D). An overview of its taxonomic status was made by Jeekel (2001).

Distribution: Paraspirobolus lucifugus is widespread in the tropics and in greenhouses from Europe (Jeekel, 2001; Enghoff et al., 2004; Korsós, 2004). In Brazil, the species has been recorded only in the coastal region from the Atlantic Forest and in urban areas. All records of the species were obtained from the literature.

Historical records: Espírito Santo: São Mateus

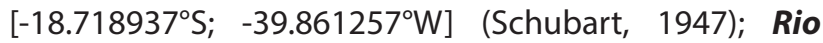
de Janeiro: Rio de Janeiro, Tijuca $\left[-22.935505^{\circ} \mathrm{S}\right.$; $-43.243112^{\circ} \mathrm{W}$ ] (Schubart, 1947); Jacarepaguá [-22.953677º ${ }^{\circ}$; $\left.-43.408759^{\circ} \mathrm{W}\right]$ (Schubart, 1947). São Paulo: Aparecida do Norte [-22.867750 $\left.{ }^{\circ} \mathrm{S} ;-45.228194^{\circ} \mathrm{W}\right]$ (Schubart, 1947); Ilhabela [-23.817663ㅇ; $-45.369504^{\circ} \mathrm{W}$ ], xi.1896, 20'5우 (Brölemann, 1902); Santos [-23.974598 ${ }^{\circ}$; $-46.307597^{\circ} \mathrm{W}$ ], ix.1896, ᄋ (Brölemann, 1902).

\section{Order Julida \\ Family Julidae}

\section{Cylindroiulus britannicus (Verhoeff, 1891)}

(Figs. 1D, 7E)

Descriptive notes: See Schubart (1942c: 250, fig. 1) and Blower (1985: 158, fig. 49).

Identification: Members of Julida are easily recognized by stipites of gnathochilarium in contact in the midline in their basal part (symphyognathous condition). The species is recognized by three pairs of setae on the anal valves (Blower, 1985: fig. 49a); opisthomerite with finger-shaped projection (Blower, 1985: fig. 49b); promerite simple; mesomerite shorter than promerite (Fig. 7E).

Distribution: The order Julida is distributed in the Holarctic region, marginally also in SE Asia and Central America (Enghoff et al., 2015). Cylindroiulus britannicus is widespread in Europe and predominantly distributed in the Northwest region (Blower, 1985). The species has been recorded in southern India, New Zealand, South Africa (Hoffman, 1999), Chile, and Argentina (Golovatch, 2014; Shelley et al., 2014). In Brazil, the species has been recorded only in urban areas in the states of São Paulo and Rio de Janeiro. All records of the species were obtained from the literature.

Historical records: São Paulo: Pirassununga [-22.005841ㅇ'; -47.424516º W], 23.vii.1940-13.ii.1941, O. Schubart coll., 20' 294 immatures (Schubart, 1942c); São

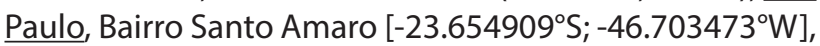
1954, O. Schubart coll., ox $0^{x} 1$ immature (Schubart, 1945a); Água Branca [-23.517304º ${ }^{\circ}$; $-46.690714^{\circ} \mathrm{W}$ ] (Schubart, 1944); Bairro do Tremembé [-23.468582º ; $-46.624367^{\circ} \mathrm{W}$ ] (Schubart, 1947); Rio de Janeiro: Itatiaia [-22.458524우 $\left.-44.562840^{\circ} \mathrm{W}\right]$ (Schubart, 1947).

\section{Cylindroiulus truncorum (Silvestri, 1896)}

(Figs. 2B, 7F)

Descriptive notes: Male, see Blower (1985: 162, fig. 51) and Korsós \& Enghoff (1990: 347, figs. 1, 5-8, 21, 30, 31).

Identification: According to the diagnosis made by Korsós \& Enghoff (1990), males of C. truncorum are recognized by promerite of gonopods slightly longer than mesomerite, without a deep mesal incision; opisthomerite with laterad bent smooth brachit, without setae or protuberances; paracoxal process long, slender and pointed, almost reaching the end of solenomerite (Blower, 1985: fig. 51; Fig. 7F).

Distribution: It is widespread in Europe and North Africa, probably by several events of introduction (Blower, 1985; Korsós \& Enghoff, 1990). The species has been reported in Hawaii, North and South America (Shelley et al., 1998; Hoffman, 1999). Cylindroiulus truncorum occurs mainly 
in synanthropic habitats such as greenhouses, gardens, and parks (Korsós \& Enghoff, 1990). In Brazil, the species has been recorded only in urban areas in São Paulo. All records of the species were obtained from the literature.

Historical records: São Paulo: São Paulo, Bairro dos Campos Elíseos [-23.5475 S; $-46.6361^{\circ} \mathrm{W}$ ], 03.iii.1944, O. Schubart \& J. Schubart coll. (Schubart, 1946a).

\section{Order Polyzoniida \\ Family Siphonotidae}

\section{Rhinotus purpureus (Pocock, 1894) (Figs. 2C, 4F, 5E-F)}

Descriptive notes: See Mauriès (1980: 1101, fig. 62) and Wesener (2014: 588, figs. 1-2, for the synonym P. malagassum).

Identification: Native species of Polyzoniida in Brazil belong to the genera Siphonotus Brandt, 1837 and Burinia Attems, 1926 (Hoffman, 1977, 1980; Enghoff et al., 2015). Males of $R$. purpureus are recognized by having sternite of anterior gonopod with two lobes carrying long setae; coxae with trichostele carrying long setae; third podomere laterally with short setae and carrying mesally a protruding channel (Mauriès, 1980: fig. 62; Wesener, 2014: fig. 2a; Fig. 5E). Posterior gonopod sternite elongated into two lobes; each one with apical setae; remaining podemeres partly fused and difficult to distinguish; tarsus elongated and apically with short claw (Wesener, 2014: fig. 2b; Fig. 5F).

Distribution: The range extension of $R$. purpureus is not known and its native area is still uncertain (Hoffman, 1999). The species has been recorded in the Neotropical region (Shelley, 1998c), West Africa, Mauritius, East Indies (Hoffman, 1999), Madagascar (Wesener, 2014), and Asia (Hoffman, 1977; Korsós, 2004). In Brazil, the species has been recorded in urban areas from Manaus and from uncertain localities in the state of Amazonas (see Hoffman et al., 1996, 2002).

Material examined: Amazonas: Manaus, sítio Vida Tropical, AM 010, km 35 [-02.759189'S; -59.920910W], 11.xi.2017, T.M. Almeida \& J.A. Rafael coll., $110^{\top 2} 2$ 오 (INPA).

Historical records: Amazonas: Manaus [-03.063877 ${ }^{\circ} \mathrm{S}$; -60.036493 W], Embrapa Amazônia Ocidental [-02.893744 ${ }^{\circ}$ S; $\left.-59.973109^{\circ} \mathrm{W}\right]$ (Hoffman et al., 2002).

\section{DISCUSSION}

According to our results, the species richness varies substantially among the federative units in Brazil, with the Southeast region concentrating $42,6 \%$ of the compiled records and 5-7 species recorded by grid (Fig. 3A-C). Some hypotheses have been made to determine wheth- er the geographic expansion of some species in Brazil is a consequence of human-assisted introduction (Schubart, 1942b, 1946a, b). For instance, Schubart (1946b) suggested that the trade route of Portuguese colonies between South America and Asia indirectly influenced the spreading of $T$. calvus throughout the Neotropical region. Based on our results, $T$. calvus has been reported only in cities with maritime commercial facilities, including the new records from Salvador, Bahia.

Introduced millipedes in the Neotropical region tend to be synanthropic (Hoffman et al., 2002). For the julidans species, some studies have reported their occurrences in synanthropic habitats in Europe and USA (Schubart, 1946a; Blower, 1985; Korsós, 1992; Proesmans \& De Smedt, 2015). The proximity of the occurrence sites of introduced millipedes in Brazil reinforces this pattern since most of the species have been observed in urban areas (Schubart, 1946a, b, 1958a, b). Based on the collecting data and the species reported here, the oldest records of introduction would be those of Paraspirobolus lucifugus from the coast of the São Paulo state in the late $19^{\text {th }}$ century (Brölemann, 1902), and posteriorly from the middle of the $20^{\text {th }}$ century for the species Prosopodesmus jacobsoni and Trachyjulus calvus in 1940 (Schubart, 1945b, 1946b) and the paradoxosomatid species Oxidus gracilis and Orthomorpha coarctata (Schubart, 1939, 1944, 1945a).

Even if the consequences of the introduction of species are poorly discussed in Brazil, some reports have been made focused on possible economic damages to poly- monocultures (Schubart, 1942b; Boock \& Lordello, 1952; Lordello, 1954; Corso, 1991; Hoffman-Campo et al., 2012). The widespread species Oxidus gracilis has been reported attacking fern (Schubart, 1942b, 1945b), seedlings of coffee (Coffea arabica L.) and lettuce (Lactuca sativa L.), yellow mombin (Spondias mombin L.), and ornamental plants (Lordello, 1954), while Orthomorpha coarctata has been found attacking seedlings of coffee and ornamental plants (Lordello, 1954), cultivars of banana (Musa L.) and vegetable gardens (Schubart, 1947), and Cylindroiulus britannicus vegetable gardens (Schubart, 1947) and lettuce seedlings (Lordello, 1954). In addition, Hoffman et al. (2002) also recorded the presence of $R$. purpureus (Siphonotidae) in tree crop plantations in Manaus, Amazonas state.

\section{CONCLUSIONS}

Although there are still gaps in knowledge about the millipede fauna in the Neotropical region, this study presents one of the first efforts for a survey of introduced species in Brazil. The findings presented here report 12 introduced species in 21 Brazilian states, with a significant portion of these records related to rural and urban areas. However, our results are far from complete since the number of known introduced species in Brazil is still incipient. In this perspective, additional studies should expand our list of millipede with the inclusion of possible other species. 


\section{ACKNOWLEDGMENTS}

We are grateful to all curators for their hospitality during the visits. This study was financially supported by grant to LFMI (2016/24248-0) from São Paulo Research Foundation (FAPESP) and RSB (2018/00103-8); by CAPES (88887.510007/2020-00) grant to RSB. ADB was supported by the grant CNPq (303903/2019-8). This study was financed in part by the Coordenação de Aperfeiçoamento de Pessoal de Nível Superior Brasil (CAPES) - Finance Code 001. TMA was supported by Fundação de Amparo à Pesquisa do Estado do Amazonas (FAPEAM) (1437/2007).

\section{AUTHORS' CONTRIBUTIONS}

LFMI, RSB, and ADB planned the manuscript. All authors analyzed the data and contributed actively in the paper writing, editing and revisions.

\section{REFERENCES}

Akkari, N. \& Enghoff, H. 2011. On some surface structures of potential taxonomic importance in families of the suborders Polydesmidea and Dalodesmidea (Polydesmida, Diplopoda). ZooKeys, 156: 1-24. D0I

Antunes, L.F.S.; Scoriza, R.N.; Silva, D.G. \& Correia, M.E.F. 2016. Production and efficiency of organic compost generated by millipede activity. Ciência Rural, 46(5): 815-819. DOI

Antunes, L.F.S.; Scoriza, R.N.; Silva, D.G. \& Correia, M.E.F. 2019. Consumo de resíduos agrícolas e urbanos pelo diplópode Trigoniulus corallinus. Nativa, 7(2): 162-168. DOI

Attems, C. 1900. Dr. Brauer's Myriopoden-Ausbeute auf den Seychellen im Jahre 1895. Zoologische Jahrbücher. Abteilung für Systematik, Geographie und Biologie der Thiere, 13:133-171.

Blower, J.G. 1985. Millipedes. Keys and notes for the identification of the species. Brill. 242p. (Synopses of the British Fauna, 35)

Bogyó, D.; Magura, T.; Simon, E. \& Tóthmérész, B. 2015. Millipede (Diplopoda) assemblages alter drastically by urbanisation. Landscape and Urban Planning, 133: 118-126. DOI

Boock, 0.J. \& Lordello, L.G.E. 1952. Diplópoda depredador de tubérculos de batatinha. Bragantia, 12: 343-348. DOI

Brölemann, H.W. 1902. Myriapodes du Musée de São Paulo. Revista do Museu Paulista, 5: 35-237. DOI

Brölemann, H.W. 1903. Myriapodes recueillis au Pará par Monsieur le Prof. E.A. Goeldi, Directeur du Musée. Zoologischer Anzeiger, 26(691): 177-191.

Brölemann, H.W. 1904. Myriapodes du Museu Paulista, Ile mémorie: Manaos. Revista do Museu Paulista, 6: 63-96. DOI

Brölemann, H.W. 1909. Os Myriapodos do Brazil. São Paulo, Museu Paulista. 94p. (Catalogos da Fauna Brazileira, 2)

Brölemann, H.W. 1920. Diplopoda. In: Alluaud, Ch. (Ed.). Voyage de Ch. Alluaud et $R$. Jeannel en Afrique Orientale 1911-1912. Résultats Scientifiques, Myriapodes. Paris, A. Schultz. v. 3: 51-298.

Brölemann, H.W. 1929. Myriapodes recueillis au Brésil par M. le Professeur Caullery, membre de I'Institut. Mémoires de la Société Zoologique de France, 26(691): 177-191.

Brunke, A.J.; O'Keefe, L.; Bahlai, C.A.; Sears, M.K. \& Hallett, R.H. 2012. Guilty by association: an evaluation of millipedes as pests of carrot and sweet potato. Journal of Applied Entomology, 136(10): 772-780. D01
Butcher, F.G. 1936. Studies of seasonal occurrence of injuries to potato tubers in western New York. Journal of Economic Entomology, 29: 486-490. D0I

Corso, I.C. 1991. Efeito de inseticidas sobre populações do piolho-de-cobra (Classe: Diplopoda; Ordem: Julida). In: EMBRAPA. Centro Nacional de Pesquisa de Soja, Londrina, PR. Resultados de Pesquisa de Soja 1985/86. Londrina. p. 91-95.

Crawford, C.S. 1992. Millipedes as model detritivores. Berichte des Naturwissenschaftlich-medizinischen Vereins in Innsbruck, 10: 227-288.

Didham, R.K.; Tylianakis, J.M.; Hutchinson, M.A.; Ewers, R.M. \& Gemmell, N.J. 2005. Are invasive species the drivers of ecological change? Trends in Ecology \& Evolution, 20(9): 470-474.

Enghoff, H. 1993. Cape Verdean millipedes (Diplopoda). Tropical Zoology, 6(1): 207-216. D0I

Enghoff, H.; Golovatch, S.I. \& Nguyen, A.D. 2004. A review of the millipede fauna of Vietnam. Arthropoda Selecta, 13(1-2): 29-43.

Enghoff, H.; Golovatch, S.l.; Short, M.; Stoev, P. \& Wesener, T. 2015. Diplopoda - taxonomic overview. In:Minelli, A. (Ed.). Treatise on Zoology - Anatomy, taxonomy, biology. The Myriapoda. Boston, Brill. p. 363-454.

Golovatch, S.I. 2014. On some new or poorly-known millipedes from Chile and Argentina (Diplopoda). Russian Entomological Journal, 23(4): 249-281.

Golovatch, S.I. \& Kime, D.R. 2009. Millipede (Diplopoda) distributions: A review. Soil Organisms, 81(3): 565-597.

Golovatch, S.I.; Geoffroy, J.J.; Mauriès, J.P. \& VandenSpiegel, D. 2007. Review of the millipede genus Glyphiulus Gervais, 1847, with descriptions of new species from Southeast Asia (Diplopoda, Spirostreptida, Cambalopsidae). Part 1: the granulatus-group. Zoosystema, 29(1): 7-49.

Golovatch, S.I.; Mikhaljova, E.V. \& Chang, H.-W. 2011. The millipede families Cryptodesmidae, Haplodesmidae, Pyrgodesmidae, Opisotretidae and Xystodesmidae in Taiwan (Diplopoda, Polydesmida). Tropical Natural History, 11(2): 119-134. https://li01.tci-thaijo.org/index.php/tnh/ article/view/102999.

Golovatch, S.I.; Geoffroy, J.J.; Mauriès, J.P. \& VandenSpiegel, D. 2012. New or poorly-known species of the millipede genus Trachyjulus Peters, 1864 (Diplopoda: Spirostreptida: Cambalopsidae). Arthropoda Selecta, 21(2): 103-129. D0I

Hoffman, R.L. 1977. On the status of Siphonotus brasiliensis Brandt, and of the diplopod family Siphonotidae (Polyzoniida). Deutsche Entomologische Zeitschrift, N.F., 24(4-5): 425-431. D0I

Hoffman, R.L. 1980. Classification of the Diplopoda. Genève, Múseum $\mathrm{d}^{\prime}$ histoire naturelle. $237 \mathrm{p}$.

Hoffman, R.L. 1994. Studies on spiroboloid millipeds. XVIII. Speleostrophus nesiotes, the first known troglobitic spiroboloid milliped, from Barrow Island, Western Australia (Diplopoda: Pachybolidae: Trigoniulinae). Myriapodologica, 3: 19-24.

Hoffman, R.L. 1999. Checklist of the millipedes of North and Middle America. Virginia Museum of Natural History, Special Publications, 8: 1-584.

Hoffman, R.L.; Golovatch, S.I.; Adis, J. \& Morais, J.W. 1996. Practical keys to the orders and families of millipedes of the Neotropical region (Myriapoda: Diplopoda). Amazoniana: Limnologia et Oecologia Regionalis Systematis Fluminis Amazonas, 14(1-2): 1-35. https://hdl.handle. net/21.11116/0000-0004-8F37-E.

Hoffman, R.L.; Golovatch, S.I.; Adis, J. \& Morais, J.W. 2002. Diplopoda. In: Adis, J. (Ed.). Amazonian Arachnida and Myriapoda: identification keys to all classes, orders, families, some genera, and lists of known terrestrial species. Sofia, Pensoft Publishers. p. 505-533.

Hoffman-Campo, C.B.; Oliveira, L.J.; Moscardi, F.; Corrêa-Ferreira, B.S. \& Corso, I.C. 2012. Pragas que atacam plântulas, hastes e pecíolos da soja. In: Hoffman-Campo, C.B.; Oliveira, L.J. \& Moscardi, F. (Eds.). Soja: manejo integrado de insetos e outros Artrópodes-praga. Brasília, DF, EMBRAPA. p. $145-212$. 
Hopkin, S.P. \& Read, H.J. 1992. The biology of Millipedes. Oxford, 0xford University Press. 233p.

Iniesta, L.F.M.; Bouzan, R.S.; Rodrigues, P.E.S.; Almeida, T.M.; Ott, R. \& Brescovit, A.D. 2020. Ecological Niche Modeling (ENM) predicting the potential invasion of the non-native millipede Oxidus gracilis (C.L. Koch, 1847) (Polydesmida, Paradoxosomatidae) in Brazilian Atlantic Forest. Annales de la Société Entomologique de France, 56(5): 387-394. D0I

Jeekel, C.A.W. 1968. On the classification and geographical distribution of the family Paradoxosomatidae (Diplopoda, Polydesmida). (Phd Thesis). University of Amsterdam, Netherlands.

Jeekel, C.A.W. 2001. Julus lucifugus Gervais, 1836, a long overlooked name for a wide-spread synanthrope millipede (Diplopoda, Spirobolida, Spirobolellidae). Myriapod Memoranda, 3: 39-43.

Jeekel, C.A.W. 2004. A bibliographic catalogue of the "Cambaloidea" (Diplopoda, Spirostreptida). Myriapod Memoranda, 7: 43-109.

Korsós, Z. 1992. Millipedes from anthropogenic habitats in Hungary (Diplopoda). Berichte des naturewissenschaftlich-medizinischen Vereins in Innsbruck, 10: 237-241.

Korsós, Z. 2004. Checklist and bibliography of millipedes (Diplopoda) of Taiwan. Collection and Research, 17: 11-32.

Korsós, Z. \& Enghoff, H. 1990. The Cylindroiulus truncorum group (Diplopoda: Julidae). Entomologica Scandinavica, 21(3): 345-360.

Korsós, Z.; Hornung, E.; Szlavecz, K. \& Kontschan, J. 2002. Isopoda and Diplopoda of urban habitats: New data to the fauna of Budapest. Annales historico-naturales Musei Nationalis Hungarici, 94: 193-208.

Kuria, J.N. \& van Eijnatten, G.L.M. 1981. Millipedes as pest of crop plants in Coast Province, Kenya. Report Cars Communication, Kenya, 1-8.

Likhitrakarn, N.; Golovatch, S.I. \& Panha, S. 2011. Revision of the Southeast Asian millipede genus Orthomorpha Bollman, 1893, with the proposal of a new genus (Diplopoda, Polydesmida, Paradoxosomatidae). Zookeys, 131: 1-161. D0I

Likhitrakarn, N.; Golovatch, S.I.; Semenyuk, I.; Efeykin, B.D. \& Panha, S. 2019. Review of the millipede genus Orthomorpha Bollman, 1893 (Diplopoda, Polydesmida, Paradoxosomatidae) in Vietnam, with several new records and descriptions of two new species. Zookeys, 898: 121-158. D0I

Lordello, L.G.E. 1954. Observação sobre alguns Diplópodos de interesse agrícola. Anais da Escola Superior de Agricultura "Luiz de Queiroz", 11: 69-76. DOI

Mauriès, J.P. 1980. Diplopodes Chilognathes de la Guadeloupe et ses dépendances. Bulletin du Muséum national d'histoire naturelle, 4e série, Section A, Zoologie, 2(4): 1059-1111.

Mesibov, R. 2012. New species of Prosopodesmus Silvestri, 1910 (Diplopoda, Polydesmida, Haplodesmidae) from Queensland, Australia. ZooKeys, 190: 33-54. DOI

Nguyen, D.A. \& Sierwald, P. 2013. A worldwide catalogue of the family Paradoxosomatidae Daday, 1889 (Diplopoda: Polydesmida). Check List, 9: 1132-1353. DOI

Nguyen, A.D.; Kórsos, Z.; Jang, K.H. \& Hwang, U.W. 2017. A revision and phylogenetic analysis of the millipede genus Oxidus Cook, 1911 (Polydesmida, Paradoxosomatidae). European Journal of Taxonomy, 293: 1-22. DOI

Nsengimana, V.; Kaplin, B.A.; Francis, F. \& Nsabimana, D. 2018. Use of soil and litter arthropods as biological indicators of soil quality in forest plantations and agricultural lands: A Review. Entomologie Faunistique Faunistic Entomology, 71: 1-12. D0I

O'Neill, R.V. \& Reichle, D.E. 1970. Urban infestation by the millipede, Oxidus gracilis (Koch). Journal of the Tennessee Academy of Science, 45: 114-115.

Potapov, A.M.; Tiunov, A.V. \& Scheu, S. 2019. Uncovering trophic positions and food resources of soil animals using bulk natural stable isotope composition. Biological Reviews, 94: 37-59. D01
Proesmans, W. \& De Smedt, P. 2015. The millipede Cylindroiulus britannicus (Verhoeff, 1891) new for the Belgian fauna (Diplopoda: Julidae). Bulletin de la Société royale belge d'Entomologie, 151(3): 239-242.

Rodrigues, P.E.S.; Costa-Schmidt, L.E.; Ott, R. \& Rodrigues, E.N.L. 2017. Influence of forest structure upon the diversity and composition of edaphic diplopods. Journal of Insect Conservation, 21(2): 297-306. D0I

Schubart, 0. 1939. Die Myriapoden des Staates Pernambuco. I. Die Familie Strongylosmidae (Diplopoda, Polydesmoidea). Zoologischer Anzeiger, 128(3-4): 77-84.

Schubart, 0. 1942a. Fauna do Estado de Pernambuco e dos Estados Limítrofes. Boletim do Museu Nacional, XIV-XVII: 20-61.

Schubart, 0. 1942b. Os Myriápodes e suas relações com a agricultura. Papéis Avulsos do Departamento de Zoologia, 22(16): 205-234.

Schubart, 0. 1942c. Diplopoda de Pirassununga I. Primeiro diplopodo Europeu encontrado no Brasil. Papéis Avulsos do Departamento de Zoologia, 2(18): 249-254.

Schubart, 0. 1944. Os Diplopodos de Pirassununga. Acta Zoologica Lilloana, 2(2): 321-440.

Schubart, 0. 1945a. Diplópodos de Monte Alegre. Papéis Avulsos do Departamento de Zoologia, São Paulo, 6(23): 283-320.

Schubart, 0. 1945b. 0s Proterospermophora do Distrito Federal (Myriapoda, Diplopoda). Arquivos do Museu Nacional, 38: 1-156.

Schubart, 0. 1946a. Uma segunda especie do genero Cylindroiulus [Diplopoda] encontrada para o Brasil. Comunicaciones Zoologicas del Museo de Historia Natural de Montevideo, 2(29): 1-5.

Schubart, 0. 1946b. "Cambalopsis nordquisti" Attems da Ásia oriental, habitante do Distrito Federal do Brasil (Diplopoda, Cambalopsidae). Revista Brasileira de Biologia, 6(3): 395-406.

Schubart, 0. 1947. 0 elemento 'Synanthropo' e estrangeiro entre os Diplopoda do Brasil. Arthropoda, 1: 23-40.

Schubart, 0. 1953. Sobre os Diplopoda dos estados do Paraná e Santa Catarina. I. Proterospermophora. Arquivos do Museu Paranaense, 10: 77-132.

Schubart, 0. 1958a. Sobre alguns Diplopoda de Mato Grosso e Goiás, Brasil e a família Spirostreptidae. Arquivos do Museu Nacional, 46: 203-252.

Schubart, 0. 1958b. Sobre alguns Diplopoda do estado do Para (Brasil), colecionados por Lauro Travassos, Gertrud Rita Kloss e Fernando D.A. Pires. Boletim do Museu Paraense Emilio Goeldi, Nova Serie, Zoologia, 16: $1-30$.

Shelley, R.M. 1998a. Occurrence of the milliped Glyphiulus granulatus (Gervais) in the Hawaiian Islands (Spirostreptida: Cambalidea: Cambalopsidae). Bishop Museum Occasional Papers, 56: 36-37.

Shelley, R.M. 1998b. Occurrence of the Milliped Trigoniulus corallinus (Gervais) on O'ahu and Kaua'i (Spirobolida: Pachybolidae: Trigoniulinae). Bishop Museum Occasional Papers, 56: 55-57.

Shelley, R.M. 1998c. Interception of the milliped Rhinotus purpureus (Pocock) at quarantine, and potential introduction of the order and family into the Hawaiian Islands (Polyzoniida: Siphonotidae). Bishop Museum Occasional Papers, 56: 54-55.

Shelley, R.M. \& Golovatch, S.I. 2000. The milliped family Haplodesmidae in the Hawaiian Islands, with records of Prosopodesmus jacobsoni from Florida and Louisiana (Diplopoda: Polydesmida). Bishop Museum Occasional Papers, 64: 48-49.

Shelley, R.M. \& Golovatch, S.I. 2011. Atlas of Myriapod Biogeography. I. Indigenous Ordinal and Supra-Ordinal Distributions in the Diplopoda: Perspectives on Taxon Origins and Ages, and a Hypothesis on the Origin and Early Evolution of the Class. Insecta Mundi, 158: 1-134.

Shelley, R.M. \& Lehtinen, P.T. 1999. Diagnoses, synonymies and occurrences of the pantropical millipeds, Leptogoniulus sorornus (Butler) and Trigoniulus corallinus (Gervais) (Spirobolida: Pachybolidae: Trigoniulinae). Journal of Natural History, 33(9): 1379-1401. DOI 
Shelley, R.M.; Bauer, S.B. \& Swift, S.F. 1998. The Milliped family Paradoxosomatidae in the Hawaiian Islands (Diplopoda: Polydesmida). Bishop Museum Occasional Papers, 56: 43-53.

Shelley, R.M.; Morrill, E.D. \& Faber, D.A. 2014. A julid milliped in Chilean Patagonia, and a compilation of ordinal representatives in South America and associated islands (Diplopoda: Julida). Insecta Mundi, 0366: 1-8.

Silvestri, F. 1910. Descrizioni preliminari di novi generi di Diplopodi. Zoologischer Anzeiger, 35(12-13): 357-364.

Suzuki, Y.; Grayston, S. \& Prescott, C. 2013. Effects of leaf litter consumption by millipedes (Harpaphe haydeniana) on subsequent decomposition depends on litter type. Soil Biology and Biochemistry, 57: 116-123. D0I
Verhoeff, K.W. 1939.Diplopoden der Insel Mauritiusund ihrezoogeographische Bedeutung. Jenaische Zeitschrift für Naturwissenschaften, 73: 37-96.

Vicente, M.C. \& Enghoff, H. 1999. The millipedes of the Canary Islands (Myriapoda: Diplopoda). Vieraea, 27: 183-204.

Wesener, T. 2014. Redescription of 'Polyzonium' malagassum, a new synonym of Rhinotus purpureus (Pocock, 1894), with notes about the occurrence of the order Polyzoniida on Madagascar (Diplopoda). Zootaxa, 3790(4): 587-594. D0I 